\title{
Pteridófitas de Santa Catarina: um olhar sobre os dados do Inventário Florístico Florestal de Santa Catarina, Brasil
}

André Luís de Gasper ${ }^{1,4}$, Alexandre Salino², Alexander C. Vibrans ${ }^{3}$, Lucia Sevegnani ${ }^{1}$, Marcio Verdi² ${ }^{2}$ Alexandre Korte ${ }^{2}$, Anita Stival dos Santos ${ }^{2}$, Susana Dreveck ${ }^{2}$, Tiago João Cadorin ${ }^{2}$, Juliane Luzia Schmitt ${ }^{2}$ e Eder Caglioni ${ }^{2}$

Recebido em 27/09/2011. Aceito em 27/03/2012

\section{RESUMO}

(Pteridófitas de Santa Catarina: um olhar sobre os dados do Inventário Florístico Florestal de Santa Catarina, Brasil): Uma das unidades federativas com melhor conhecimento de sua flora, Santa Catarina é o primeiro estado a concluir o Inventário Florístico Florestal na atualidade. Coberto por Floresta Ombrófila Densa, Floresta Ombrófila Mista, Floresta Estacional Decidual e formações associadas, possui grande riqueza de espécies. Este trabalho visa apresentar as espécies de pteridófitas coletadas nas 563 unidades amostrais visitadas. Ao todo 324 espécies foram registradas, das quais 300 são samambaias e 24, licófitas. Estas pertencem a 29 famílias e 94 gêneros, sendo as famílias com maior riqueza específica Polypodiaceae (48 espécies), Pteridaceae (42) e Dryopteridaceae (38). Os gêneros com maior diversidade de espécies foram Asplenium e Thelypteris, com 27 espécies, seguido por Blechnum com 15. Destacam-se ainda 75 espécies consideradas endêmicas para o bioma. 18 registros novos para a flora de Santa Catarina foram feitos. A distribuição por região fitoecológica é a que segue: 288 espécies para a Floresta Ombrófila Densa (128 exclusivas desta região fitoecológica), 177 para a Floresta Ombrófila Mista (30 exclusivas) e 57 para a Floresta Estacional Decidual (três exclusivas). Foram registradas ainda 17 espécies para a restinga. Destaca-se a importância do registro de Asplenium lacinulatum, espécie coletada em área de intensa exploração imobiliária e novo registro para Santa Catarina e Alansmia senilis primeiro registro para o Sul do Brasil.

Palavras-chave: samambaia, Mata Atlântica, floresta ombrófila, floresta estacional, restinga

\begin{abstract}
(Ferns and Fern allies from Santa Catarina State: a "look at the data" from Santa Catarina Floristic Forest Inventory, Brazil). Santa Catarina is the first Brazilian state to complete its Forest and Floristic Inventory, and is considered one of the states with a well-known flora. This region is covered by evergreen tropical rain forest, Araucaria forest, seasonal deciduous forest and associated ecosystems, and shows high species richness. This paper presents a list of ferns collected in 563 sampling units. Altogether, 324 species were recorded (300 ferns and 24 lycophytes), which belong to 29 families and 94 genera. The most diverse families are Polypodiaceae (49 species), Pteridaceae (42), and Dryopteridaceae (38). Asplenium and Thelypteris are the most diverse genera, with 27 species each, followed by Blechnum (15). Of importance, too, are the 75 species considered endemic to the biome. Eighteen new records for the flora of Santa Catarina were made. The distribution of species according to the vegetation type is the following: 288 species occur in evergreen tropical rainforest (of which 128 are exclusive to this vegetation type); 177 species in Araucaria forest (30 exclusively) and 57 species in seasonal deciduous forest ( 3 exclusively). In addition, 17 species were recorded for restinga. A collection of Asplenium lacinulatum, from an area of intense development, is first record for Santa Catarina, and another of Alansmia senilis is the first record for the South Region of Brazil.
\end{abstract}

Key words: Atlantic Forest, ombrophilous forest, seasonal forest, restinga

\footnotetext{
1 Universidade Regional de Blumenau, Herbário Dr. Roberto Miguel Klein, Blumenau, SC, Brasil

2 Universidade Federal de Minas Gerais, Departamento de Botânica, Belo Horizonte, MG, Brasil

3 Universidade Regional de Blumenau, Departamento de Engenharia Florestal, Blumenau, SC, Brasil

4 Autor para correspondência: algasper@gmail.com
} 


\section{Introdução}

Atualmente, as pteridófitas estão separadas em dois grupos monofiléticos, as licófitas e as samambaias (Pryer et al. 2001; Pryer et al. 2004; Smith et al. 2006) e englobariam aproximadamente 12 mil espécies no mundo (Moran 2008). Nas Américas, Windisch (1992) estima que ocorram cerca de 3.250 espécies de pteridófitas, das quais 30\% podem ser encontradas no território brasileiro. Para o Brasil, 1176 espécies estão registradas no Catálogo de Plantas e Fungos do Brasil (Forzza et al. 2010). Para a Mata Atlântica Salino \& Almeida (2009) registraram 840 espécies.

Com elevada riqueza em Santa Catarina, as pteridófitas ocupam vários ambientes em variadas formas (Page 1979a, 1979b; Senna \& Waechter 1997; Windisch 2002; Gasper \& Sevegnani 2010) no bioma Mata Atlântica, o mais ameaçado no país (Morellato \& Haddad 2000). Este cobre todo o território catarinense em diversas regiões fitoecológicas como a Floresta Ombrófila Densa, Floresta Ombrófila Mista, Floresta Estacional Decidual e as formações associadas como pioneira de influência flúvio-marinha (manguezais) e de influência marinha (restingas), ricas em espécies (Klein 1978; IBGE 1992; Leite \& Klein 1990).

Estado pioneiro nos estudos de sua flora (Reitz 1965), Santa Catarina registra 402 espécies de pteridófitas de acordo com Prado \& Sylvestre (2010), e boa parte do conhecimento acerca desse grupo no Sul do Brasil pode ser encontrado já nas obras de Sehnem (1967a-c; 1968a, b; 1970a, b; 1971; 1972; 1974; 1978; 1979a-g; 1984). Mesmo assim, novos registros ainda são feitos (Gasper \& Sevegnani 2010).

Após os trabalhos desenvolvidos pelo herbário Barbosa Rodrigues e registrados por Reitz (1965), um novo esforço amostral vem sendo feito em Santa Catarina. O Inventário Florístico Florestal de Santa Catarina (IFFSC) visa compreender melhor a qualidade dos remanescentes florestais do estado, além de contribuir para o conhecimento da Flora atual de Santa Catarina (Vibrans et al. 2010).

Com base nas coletas recentes do IFFSC este trabalho pretende apresentar a listagem das espécies de pteridófitas encontradas e suas formações florestais de ocorrência, além de apresentar novos registros para o estado.

\section{Material e métodos}

Durante o período de novembro de 2007 a julho de 2011, 563 unidades amostrais (UA), figura 1, foram visitadas em todo o estado de Santa Catarina (Vibrans et al. 2010), com altitudes que variavam de 2 a 1700 metros. Além de estudos fitossociológicos como os apresentados por Vibrans et al. (2011) e Gasper et al. (2011), material fértil foi coletado tanto nos fragmentos quanto em eventuais paradas em pontos ao longo do deslocamento entre os fragmentos visitados. Neste trabalho apenas as pteridófitas foram analisadas.

Santa Catarina é considerada por Nimer (1971) como pertencente a zona temperada, região de grande formação de nuvens e consequentemente de chuva. O mesmo autor considera a região privilegiada pelas suas altitudes e pelo regime de precipitação, cujo total anual varia de 1250 a 2000 $\mathrm{mm}$, o que faz com que não haja excesso nem carência de água, nem uma estação seca. Duas estações são bem distintas: o inverno frio e verão moderadamente quente (Klein 1984). A região do todo o estado pode ser considerada como de clima superúmido (Nimer 1971).

Todo o material coletado foi devidamente prensado conforme as metodologias vigentes (Fidalgo \& Bononi 1984) e as exsicatas foram tombadas no herbário FURB, com duplicatas enviadas aos herbários BHCB, MBM e RB. A identificação deu-se com consulta a especialistas e bibliografia pertinente. Os nomes dos autores seguem Pichi Sermolli (1996) e a classificação para famílias de samambaias Smith et al. (2006) e licófitas Kramer \& Green (1990).

Foram registrados os hábitos das espécies: erva terrícola, rupícola, epífita, lianescente/escandente, feto arborescente ou hidrófita. As espécies também foram classificadas conforme a distribuição geográfica (adaptado de Moran \& Smith 2001; Parris 2001) e regiões fitoecológicas ocorrente (conforme Klein,1978).

\section{Resultados e discussão}

Foram identificadas 324 espécies de pteridófitas (Tab. 1), sendo 300 samambaias e 24 licófitas. Estas pertencem a 29 famílias e 94 gêneros, sendo as famílias com maior riqueza específica Polypodiaceae (48 espécies), Pteridaceae (42) e Dryopteridaceae (38) (Fig. 2). Os gêneros com maior número de espécies foram Asplenium e Thelypteris, com 27 espécies, seguido por Blechnum com 15. Destacam-se ainda 75 espécies consideradas endêmicas para a Mata Atlântica, de acordo com Salino \& Almeida (2009) (Tab. 1). Quanto a distribuição geográfica $89 \%$ ocorrem apenas nas Américas, sendo destas $26,4 \%$ endêmicas a região sudeste e sul do Brasil (Fig. 3).

Este elevado endemismo das espécies para o bioma deve-se em parte à totalidade da cobertura do estado de Santa Catarina ser de Mata Atlântica, relativamente ainda conservada (cobertura estimada em 30\%), principalmente na região costeira. Este valor é próximo (24,5\%) do encontrado por Kluge \& Kessler (2006) que sugerem que o histórico das espécies (migração) e mudanças climáticas possam ser grandes responsáveis pelo endemismo.

Até o presente, este estudo registrou 79,1\% das espécies citadas pela Flora do Brasil (Forzza et al. 2010). Ainda registraram-se 18 novos táxons para Santa Catarina, a saber: Alansmia senilis (Fée) Moguel \& M.Kessler, Asplenium formosum Willd., Asplenium lacinulatum Schrad., Diplazium leptocarpon Fée, Doryopteris varians Sm., Elaphoglossum longifolium (C. Presl) J. Sm., Huperzia comans (Herter ex Nessel) B. Øllg. \& P.G. Windisch, Huperzia mollicoma (Spring) Holub, Hymenophyllum delicatulum Sehnem, Melpomene flabelliformis (Poir.) A.R. Sm. \& R.C. 


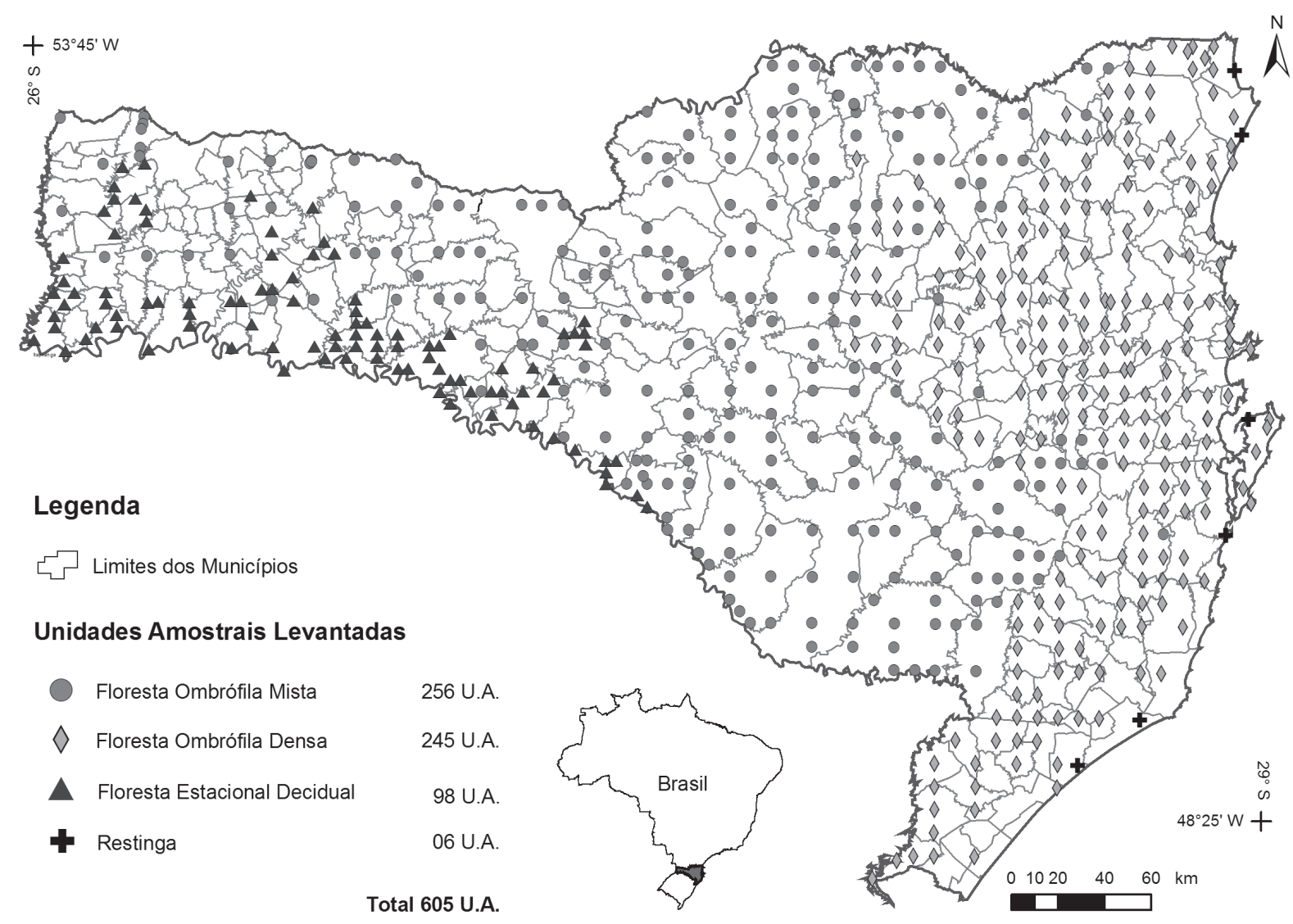

Figura 1. Localização das Unidades Amostrais (UA’s) do Inventário Florístico Florestal de Santa Catarina e suas respectivas regiões fitoecológicas.

Moran, Polyphlebium diaphanum (Kunth) Ebihara \& Dubuisson, Sticherus lanuginosus (Fée) Nakai, Tectaria pilosa (Fée) R.C. Moran, Tectaria vivipara Jermy \& T.G. Walker, Thelypteris burkartii Abbiatti, Thelypteris decurtata (Link) de la Sota, Thelypteris hatschbachii A.R. Sm. e Thelypteris maxoniana A. R. Sm..

Pode-se destacar a presença de Dicksonia sellowiana, que consta da lista das ameaçadas de extinção de acordo com a Instrução Normativa MMA 06, de 23 de setembro de 2008 (Ministério do Meio Ambiente 2008). Esta espécie apresentou distribuição heterogênea, ocorrendo por vezes em grandes manchas com mais de 907 indivíduos por hectare a manchas com indivíduos bem mais esparsos (Mantovani 2004; Gasper et al. 2011). Em 8 unidades amostrais a espécie demonstrou comportamento de monodominância ocorrendo com mais de $50 \%$ de dominância.

A perda de habitat, seguida da invasão de espécie exóticas, representa uma grande ameaça à biodiversidade (Wilcove et al. 1998); por esta razão, a conservação mesmo de pequenos remanescentes florestais como os das regiões litorâneas de Santa Catarina pode contribuir para a preservação de espécies de pteridófitas, como no caso de Asplenium lacinulatum. Esta espécie foi registrada apenas a partir decoletas do IFFSC, apesar do grande esforço amostral já realizado por outros pesquisadores (Reitz 1965). Contudo,
A. lacinulatum foi coletado em área de forte expansão imobiliária, região de Floresta Ombrófila Densa de Terras Baixas, e corre risco de ser localmente extinto devido a esta expansão, como já citado por Sylvestre (2001) para outras populações.

A grande plasticidade que o grupo apresenta pode ser observada nos seus hábitos e nos ambientes preferenciais ocupados, sendo que, do total de 324 espécies, 227 (70\%) são terrícolas, $138(42,6 \%)$ epífitas, $88(27,1 \%)$ rupícolas, $8(2,4 \%)$ são fetos arborescentes, $9(2,8 \%)$ são escandentes e três $(0,9 \%)$ é hidrófita. Muitas espécies ainda podem apresentar mais de um hábito, sendo 215 exclusivas de um único. Algumas espécies podem ser consideradas epífitos acidentais, já que raramente são vistas desta forma, mas foram aqui contadas como Diplazium cristatum, Diplazium riedelianum, Blechnum sampaioanum, entre outros.

Quanto às espécies exóticas, registram-se Deparia petersenii (Kunze) M. Kato, Thelypteris dentata (Forssk.) E.P. St. John, Pteris longifolia L. e Macrothelypteris torresiana (Gaudich.) Ching, espécies de ocorrência subespontânea em toda a América. Deparia petersenii pode ser encontrada mesmo no interior de fragmentos grandes e conservados, enquanto as outras três espécies estão mais associadas a áreas ruderais.

Considerando o conjunto de áreas amostradas de cada região fitoecológica, na Floresta Ombrófila Densa foram re- 


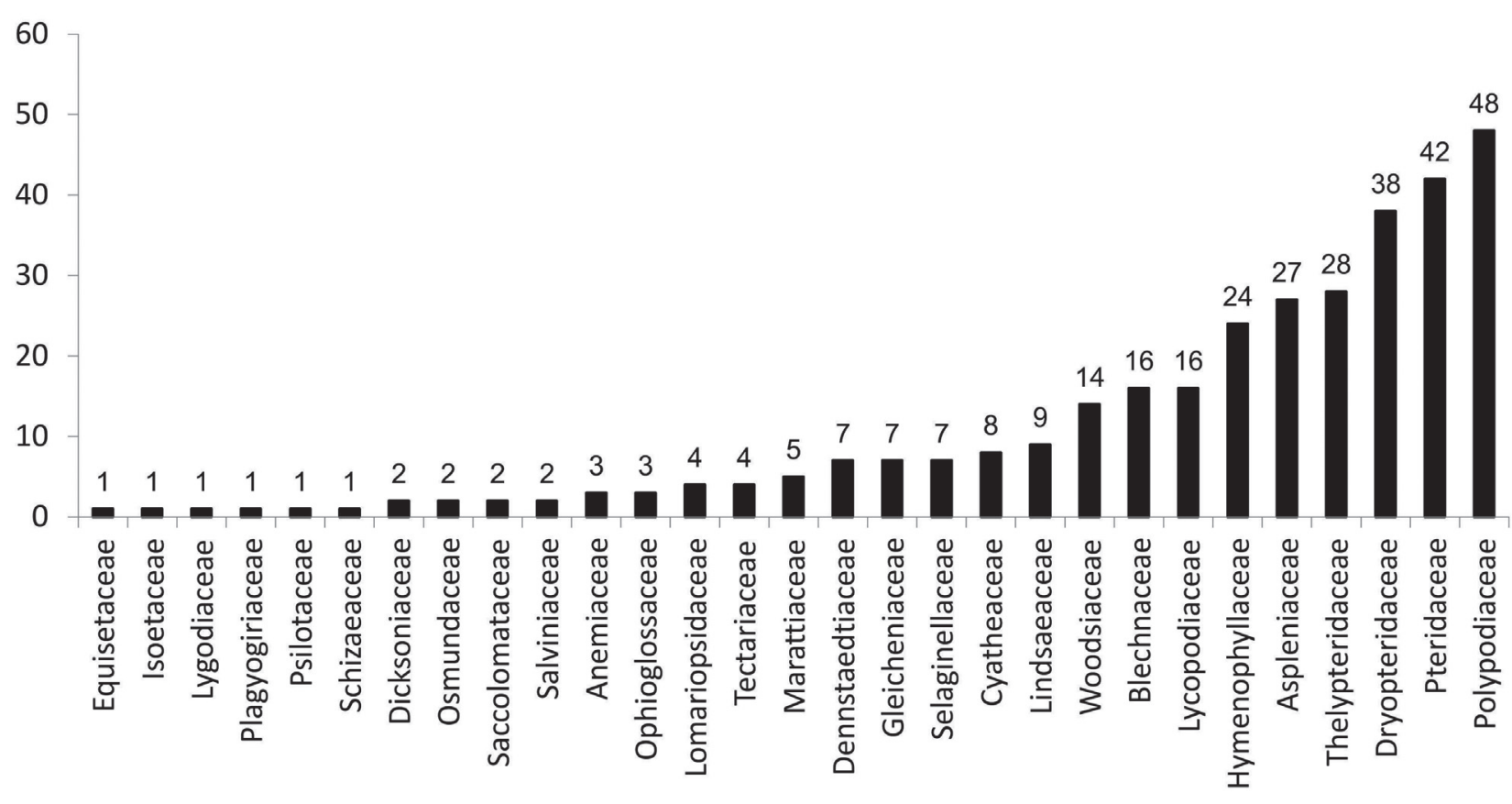

Figura 2. Distribuição das espécies de pteridófitas entre as 27 famílias registradas no Inventário Florístico Florestal de Santa Catarina

gistradas 288 espécies, sendo 128 exclusivas desta formação; na Floresta Ombrófila Mista foram 177, com 30 exclusivas; na Floresta Estacional Decidual foram 57 (3 exclusivas) e, nas restingas, 17 espécies (Fig. 4)

Devido às grandes variações geomorfológicas, à grande diversidade de formações geológicas e à complexidade de atuação dos agentes morfogenéticos, a região Sul do Brasil e consequentemente Santa Catarina torna-se bastante peculiar na sua fisionomia que acaba exercendo ponderável influência na compartimentação do clima e da vegetação (Leite \& Klein 1990), o que favorece o desenvolvimento das pteridófitas em todo o território catarinense.

Cada formação possui características próprias como espécies típicas e ambientes característicos. A maior riqueza de espécies encontra-se na Floresta Ombrófila Densa, como esperado pode ser devido à sua grande diversidade geológica (Moran 1995; Martinelli 2007) e heterogeneidade ambiental. Os índices pluviométricos são altos e bem distribuídos ao longo do ano (Nimer 1971), o que possibilita o desenvolvimento de uma pujante vegetação, principalmente das pteridófitas da ordem Polypodiales, que teriam divergido justamente neste ambiente sombreado e úmido (Schneider et al. 2004).

A Floresta Ombrófila Mista, região fitoecológica que cobre a maior parte do território catarinense (Klein 1978), apresenta elevados valores de diversidade, mesmo com atividade de pastoreio (Vibrans et al. 2011), que afeta diretamente as espécie terrícolas (Sampaio \& Guarino 2007). Blume et al. (2010) em trabalho com área amostral de 1 ha, registraram 42 espécies em um trecho de Floresta Ombrófila Mista do Rio Grande do Sul. Já Kozera et al. (2006) registraram 46 terrícolas e rupícolas e Dittrich et al. (1999) registraram 21 espécie de epífitos no Paraná e Bittencourt et al. (2004) em estudo de estrutura que identificou mais de 31 mil indivíduos por hectare para esta região fitoecológica.

Mesmo para a Floresta Estacional Decidual, o número de espécies é elevado. Nesta região com presença reduzida de epífitos (Klein 1972, 1978, Leite \& Klein 1990), havia até o momento o registro de apenas 38 espécies para toda a região fitoecológica no Bioma Mata Atlântica (Salino \& Almeida 2009); mais recentemente, Lehn et al. (2009) registraram 56 espécies para dois fragmentos na Floresta Estacional Decidual no Rio Grande do Sul.

Algumas espécies citadas por Sehnem (1967a-c; 1968a, b; 1970a, b; 1971; 1972; 1974; 1978; 1979a-g; 1984), Fuchs-Eckert (1986) e Klein (1980a, b), não foram encontradas e merecem destaque como as das famílias aquáticas Marsileaceae e Salviniaceae, pelo fato das coletas do IFFSC não abrangerem regiões de campos e banhados. Coletas nestes ambientes se fazem necessárias, haja vista que os campos sulinos encontram-se entre os ecossistemas altamente ameaçados pela pecuária e pelas queimadas.

A grande diversidade encontrada por este estudo demonstra a importância de levantamentos florísticos, uma vez que o registro da biodiversidade é fundamental para a sua proteção, além de prover dados precisos para estudos de distribuição e modelagem (Hortal et al. 2008).

A Floresta Estacional Decidual em Santa Catarina ocupa pequena área e está carente de maiores unidades de conservação, tanto em Santa Catarina como em todo o Neotrópico (Sastre \& Lobo 2009), colocando as espécies que ali ocorrem sob constante ameaça. 
Tabela 1. Espécies de pteridófitas amostradas no Inventário Florístico Florestal de Santa Catarina, agrupadas por família conforme Smith et al. (2006) e Kramer \& Green (1990). Hábito (H): A = feto arborescente; $\mathrm{Et}$ = erva terrícola; $\mathrm{R}=$ rupícola; Ep = epífita Es = escandente; $\mathrm{H}=$ hidrófita. As regiões fitoecológicas foram classificadas como: D: Floresta Ombrófila Densa, M: Floresta Ombrófila Mista, D: Floresta Estacional Decidual e R: restinga. Os coletores são A.L. de Gasper, M. Verdi, S. Dreveck, A. Stival-Santos, A. Korte, T.J. Cadorin e J.L. Schmitt

\begin{tabular}{|c|c|c|c|c|c|}
\hline Família & Espécie & Distribuição & Fitofisionomia & Habitat & Voucher \\
\hline \multirow[t]{3}{*}{ Anemiaceae } & Anemia phyllitidis (L.) Sw. & América Tropical & $\mathrm{D} / \mathrm{M} / \mathrm{E}$ & $\mathrm{Ep} / \mathrm{Et} / \mathrm{R}$ & Gasper 1351 \\
\hline & Anemia raddiana Link & Endêmica do Sul e Sudeste & $\mathrm{D} / \mathrm{E}$ & Et & Gasper 1168 \\
\hline & Anemia tomentosa (Savigny) Sw. & América Tropical & $\mathrm{D} / \mathrm{M} / \mathrm{E}$ & Et & Korte 7073 \\
\hline \multirow[t]{27}{*}{ Aspleniaceae } & Asplenium abscissum Willd. & América Tropical & $\mathrm{D}$ & $\mathrm{Ep} / \mathrm{Et} / \mathrm{R}$ & Verdi 3135 \\
\hline & Asplenium alatum Humb. \& Bonpl. ex Willd. & América Tropical & $\mathrm{D} / \mathrm{M}$ & $\mathrm{Ep} / \mathrm{Et}$ & Gasper 1062 \\
\hline & Asplenium auriculatum $\mathrm{Sw}$. & América Tropical & $\mathrm{D} / \mathrm{M}$ & Ep & Gasper 1517 \\
\hline & Asplenium brasiliense Sw. & América do Sul & $\mathrm{D} / \mathrm{E}$ & $\mathrm{Et} / \mathrm{R}$ & Gasper 1680 \\
\hline & Asplenium claussenii Hieron. & América Tropical & $\mathrm{D} / \mathrm{M} / \mathrm{E}$ & $\mathrm{Ep} / \mathrm{Et} / \mathrm{R}$ & Dreveck 173 \\
\hline & Asplenium feei Kunze ex Fée & América Tropical & $\mathrm{D}$ & $\mathrm{Ep} / \mathrm{Et}$ & Cadorin 668 \\
\hline & Asplenium formosum Willd. & Pantropical & $\mathrm{D}$ & Ep & Korte 443 \\
\hline & Asplenium gastonis Fée & América do Sul & $\mathrm{D} / \mathrm{M} / \mathrm{E}$ & $\mathrm{Ep} / \mathrm{Et} / \mathrm{R}$ & Gasper 1724 \\
\hline & Asplenium harpeodes Kunze & América Tropical & $\mathrm{D} / \mathrm{M}$ & $\mathrm{Ep} / \mathrm{Et}$ & Gasper 1740 \\
\hline & Asplenium inaequilaterale Willd. & Pantropical & $\mathrm{D} / \mathrm{M} / \mathrm{E}$ & $\mathrm{Ep} / \mathrm{Et} / \mathrm{R}$ & Stival-Santos 476 \\
\hline & Asplenium incurvatum Fée & Endêmica do Brasil & $\mathrm{D} / \mathrm{M}$ & $\mathrm{Ep} / \mathrm{Et} / \mathrm{R}$ & Stival-Santos 605 \\
\hline & Asplenium kunzeanum Klotzsch ex Rosenst. & Endêmica do Brasil & $\mathrm{D} / \mathrm{M}$ & $\mathrm{Ep} / \mathrm{Et} / \mathrm{R}$ & Schmitt 338 \\
\hline & Asplenium lacinulatum Schrad. & Endêmica do sul do Brasil & $\mathrm{D}$ & Et & Gasper 2372 \\
\hline & Asplenium martianum C. Chr. & Endêmica do Brasil & $\mathrm{D} / \mathrm{M}$ & $\mathrm{Ep} / \mathrm{Et}$ & Gasper 1887 \\
\hline & Asplenium mucronatum C. Presl & Endêmica do Brasil & $\mathrm{D}$ & Ep & Gasper 2211 \\
\hline & Asplenium oligophyllum Kaulf. & América do Sul & $\mathrm{D}$ & $\mathrm{Ep} / \mathrm{Et} / \mathrm{R}$ & Cadorin 654 \\
\hline & Asplenium pseudonitidum Raddi & Endêmica do Sul e Sudeste & $\mathrm{D} / \mathrm{M}$ & $\mathrm{Ep} / \mathrm{Et}$ & Schmitt 476 \\
\hline & Asplenium pteropus Kaulf. & América Tropical & $\mathrm{D}$ & Ep & Korte 422 \\
\hline & Asplenium raddianum Gaudich. & América do Sul & $\mathrm{D}$ & Ep & Gasper 2473 \\
\hline & Asplenium radicans $\mathrm{L}$. & América Tropical & $\mathrm{D}$ & Et & Korte 813 \\
\hline & Asplenium scandicinum Kaulf. & América do Sul & $\mathrm{D} / \mathrm{M}$ & $\mathrm{Ep} / \mathrm{Et}$ & Verdi 2380 \\
\hline & Asplenium serra Langsd. \& Fisch. & América Tropical & $\mathrm{D} / \mathrm{M}$ & $\mathrm{Ep} / \mathrm{Et}$ & Gasper 2424 \\
\hline & Asplenium serratum $\mathrm{L}$. & América Tropical & $\mathrm{D} / \mathrm{M}$ & $\mathrm{Ep} / \mathrm{Et}$ & Korte 5012 \\
\hline & Asplenium squamosum $\mathrm{L}$. & América Tropical & M & Et & Dreveck 875 \\
\hline & Asplenium triquetrum N. Murak. \& R.C. Moran & América do Sul & $\mathrm{D} / \mathrm{M}$ & $\mathrm{Ep} / \mathrm{Et} / \mathrm{R}$ & Schmitt 1702 \\
\hline & Asplenium ulbrichtii Rosenst. & América do Sul & M & Et & Gasper 1798 \\
\hline & Asplenium uniseriale Raddi & América Tropical & $\mathrm{D} / \mathrm{M}$ & $\mathrm{Ep} / \mathrm{Et} / \mathrm{R}$ & Gasper 2471 \\
\hline \multirow[t]{12}{*}{ Blechnaceae } & Blechnum australe subsp. auriculatum (Cav.) de la Sota & América do Sul & $\mathrm{M} / \mathrm{E}$ & Et & Gasper 1965 \\
\hline & Blechnum austrobrasilianum de la Sota & América do Sul & $\mathrm{E}$ & Et & Stival-Santos 654 \\
\hline & $\begin{array}{l}\text { Blechnum binervatum subsp. acutum (Desv.) R.M. Tryon } \\
\text { \& Stolze }\end{array}$ & América Tropical & $\mathrm{D} / \mathrm{M} / \mathrm{E}$ & $\begin{array}{l}\mathrm{Ep} / \mathrm{Et} / \\
\mathrm{Es} / \mathrm{R}\end{array}$ & Schmitt 156 \\
\hline & Blechnum brasiliense Desv. & América Tropical & $\mathrm{D} / \mathrm{M} / \mathrm{E} / \mathrm{R}$ & Et & Verdi 3427 \\
\hline & Blechnum cordatum (Desv.) Hieron. & América do Sul & $\mathrm{D} / \mathrm{M}$ & Et & Korte 752 \\
\hline & Blechnum divergens Mett. & América Tropical & $\mathrm{D} / \mathrm{M}$ & $\mathrm{Et} / \mathrm{R}$ & Schmitt 1130 \\
\hline & Blechnum gracile Kaulf. & América Tropical & $\mathrm{D}$ & Et & Schmitt 2171 \\
\hline & Blechnum lehmannii Hieron. & América Tropical & $\mathrm{D}$ & Et & Verdi 3146 \\
\hline & Blechnum occidentale $\mathrm{L}$. & América Tropical & $\mathrm{D} / \mathrm{M} / \mathrm{E} / \mathrm{R}$ & Et & Korte 6130 \\
\hline & Blechnum penna-marina (Poir.) Kuhn & Anfipacífico & M & Et & Gasper 2090 \\
\hline & Blechnum polypodioides Raddi & América Tropical & $\mathrm{D} / \mathrm{M}$ & $\mathrm{Ep} / \mathrm{Et} / \mathrm{R}$ & Stival-Santos 1766 \\
\hline & Blechnum sampaioanum Brade & Endêmica do Brasil & $\mathrm{D} / \mathrm{M}$ & $\mathrm{Ep} / \mathrm{Et}$ & Dreveck 1695 \\
\hline
\end{tabular}


Tabela 1. Continuação

\begin{tabular}{|c|c|c|c|c|c|}
\hline Família & Espécie & Distribuição & Fitofisionomia & Habitat & Voucher \\
\hline & Blechnum schomburgkii (Klotzsch) C. Chr. & Endêmica do Brasil & $\mathrm{D} / \mathrm{M}$ & Et & Gasper 1631 \\
\hline & Blechnum serrulatum Rich. & América Tropical & $\mathrm{D} / \mathrm{R}$ & Et & Gasper 2347 \\
\hline & Blechnum squamipes (Hieron.) M. Kessler \& A.R. Sm. & América do Sul & M & Et & Gasper 2959 \\
\hline & Salpichlaena volubilis (Kaulf.) J. Sm. & América Tropical & $\mathrm{D}$ & Es & Cadorin 67 \\
\hline \multirow[t]{8}{*}{ Cyatheaceae } & Alsophila capensis subsp. polypodioides (Sw.) D.S. Conant & Endêmica do Sul e Sudeste & M & $\mathrm{R}$ & Gasper 2961 \\
\hline & Alsophila setosa Kaulf. & América do Sul & $\mathrm{D} / \mathrm{M} / \mathrm{E}$ & A & Verdi 474 \\
\hline & Cyathea atrovirens (Langsd. \& Fisch.) Domin & América do Sul & $\mathrm{D} / \mathrm{R}$ & A & Verdi 2419 \\
\hline & Cyathea corcovadensis (Raddi) Domin. & Endêmica do Brasil & $\mathrm{D} / \mathrm{M}$ & A & Gasper 890 \\
\hline & Cyathea delgadii Sternb. & América Tropical & $\mathrm{D} / \mathrm{M}$ & A & Dreveck 916 \\
\hline & Cyathea gardneri Hook. & Endêmica do Sul e Sudeste & $\mathrm{D} / \mathrm{M}$ & A & Stival-Santos 1125 \\
\hline & Cyathea hirsuta C. Presl & Endêmica do Brasil & $\mathrm{D}$ & A & Stival-Santos 804 \\
\hline & Cyathea phalerata Mart. & Endêmica do Brasil & $\mathrm{D} / \mathrm{M} / \mathrm{R}$ & A & Verdi 457 \\
\hline \multirow[t]{2}{*}{ Dennstaedtiaceae } & Dennstaedtia cicutaria (Sw.) T. Moore & América Tropical & $\mathrm{D} / \mathrm{M}$ & Et & Verdi 182 \\
\hline & Dennstaedtia dissecta (Sw.) T. Moore & América do Sul & $\mathrm{D} / \mathrm{E}$ & $\mathrm{Et} / \mathrm{R}$ & Dreveck 717 \\
\hline \multirow[t]{5}{*}{ Dennstaedtiaceae } & Dennstaedtia globulifera (Poir.) Hieron. & América Tropical & $\mathrm{D} / \mathrm{M} / \mathrm{E}$ & $\mathrm{Et} / \mathrm{R}$ & Dreveck 757 \\
\hline & Dennstaedtia obtusifolia (Willd.) T. Moore & América do Sul & M & Et & Gasper 1650 \\
\hline & Histiopteris incisa (Thunb.) J. Sm. & Pantropical & $\mathrm{D} / \mathrm{M}$ & Et & Gasper 1406 \\
\hline & Hypolepis mitis Kunze ex Kuhn & Endêmica do Brasil & $\mathrm{D} / \mathrm{M}$ & Et & Verdi 123 \\
\hline & Pteridium arachnoideum (Kaulf.) Maxon & América Tropical & $\mathrm{D}$ & Et & Gasper 2445 \\
\hline \multirow[t]{2}{*}{ Dicksoniaceae } & Dicksonia sellowiana Hook. & América Tropical & $\mathrm{D} / \mathrm{M} / \mathrm{E}$ & A & Verdi 181 \\
\hline & Lophosoria quadripinnata (J.F. Gmel.) C. Chr. & América Tropical & $\mathrm{D} / \mathrm{M}$ & Et & Verdi 2105 \\
\hline \multirow[t]{23}{*}{ Dryopteridaceae } & Bolbitis serratifolia Schott & América tropical & $\mathrm{D}$ & Et & Dreveck 1904 \\
\hline & Ctenitis anniesii (Rosenst.) Copel. & Endêmica do sul do Brasil & $\mathrm{D} / \mathrm{M}$ & Et & Gasper 933 \\
\hline & Ctenitis falciculata (Raddi) Ching & América do Sul & $\mathrm{D} / \mathrm{M} / \mathrm{E}$ & $\mathrm{Et} / \mathrm{R}$ & Dreveck 157 \\
\hline & Ctenitis laetevirens (Rosenst.) Salino \& Morais & Endêmica do sul do Brasil & $\mathrm{D} / \mathrm{M}$ & Et & Gasper 1688 \\
\hline & Ctenitis pedicellata (Christ.) Copel. & Endêmica do Brasil & $\mathrm{D} / \mathrm{M} / \mathrm{E}$ & Et & Schmitt 341 \\
\hline & Ctenitis submarginalis (Langsd. \& Fisch.) Ching & América tropical & $\mathrm{D} / \mathrm{M} / \mathrm{E}$ & Et & Gasper 1350 \\
\hline & Didymochlaena truncatula (Sw.) J. Sm. & Pantropical & $\mathrm{D} / \mathrm{M} / \mathrm{E}$ & Et & Gasper 1061 \\
\hline & Dryopteris wallichiana (Spreng.) Hyl. & Pantropical & M & Et & Verdi 3860 \\
\hline & Elaphoglossum edwallii Rosenst. & Endêmica do Sul e Sudeste & M & $\mathrm{R}$ & Gasper 2897 \\
\hline & Elaphoglossum gayanum (Fée) T. Moore & América tropical & $\mathrm{D} / \mathrm{M}$ & $\mathrm{Ep} / \mathrm{R}$ & Gasper 1124 \\
\hline & Elaphoglossum glaziovii (Fée) Brade & América do Sul & $\mathrm{D} / \mathrm{M}$ & $\mathrm{Ep} / \mathrm{Et} / \mathrm{R}$ & Dreveck 167 \\
\hline & Elaphoglossum iguapense Brade & Endêmica do Brasil & $\mathrm{D}$ & $\mathrm{Ep} / \mathrm{R}$ & Verdi 4925 \\
\hline & Elaphoglossum lingua (C. Presl) Brack. & América Tropical & $\mathrm{D}$ & $\mathrm{Ep} / \mathrm{R}$ & Cadorin 50 \\
\hline & Elaphoglossum longifolium (C. Presl) J. Sm. & América tropical & $\mathrm{D}$ & Ep & Schmitt 1687 \\
\hline & Elaphoglossum luridum (Fée) Christ & América do Sul & $\mathrm{D}$ & $\mathrm{Ep} / \mathrm{Et}$ & Schmitt 3 \\
\hline & Elaphoglossum macahense (Fée) Rosenst. & Endêmica do Brasil & $\mathrm{D}$ & Ep & Schmitt 1043 \\
\hline & Elaphoglossum nigrescens (Hook.) T. Moore & América tropical & $\mathrm{D}$ & Ep & Korte 5000 \\
\hline & Elaphoglossum paulistanum Rosenst. & Endêmica do Sul e Sudeste & $\mathrm{D} / \mathrm{M}$ & Ep & Gasper 1644 \\
\hline & Elaphoglossum squamipes (Hook.) T. Moore & América tropical & $\mathrm{D}$ & Ep & Cadorin 2867 \\
\hline & Elaphoglossum strictum (Raddi) T. Moore & América Tropical & $\mathrm{D}$ & Ep & Schmitt 118 \\
\hline & Elaphoglossum ulei $\mathrm{H}$. Christ & Endêmica do Sul e Sudeste & M & $\mathrm{R}$ & Gasper 2958 \\
\hline & Elaphoglossum vagans (Mett.) Hieron. & Endêmica do Sul e Sudeste & $\mathrm{D} / \mathrm{M}$ & $\mathrm{Ep} / \mathrm{Et}$ & Cadorin 202 \\
\hline & Lastreopsis amplissima (C. Presl) Tindale & América do Sul & $\mathrm{D} / \mathrm{M}$ & $\mathrm{Ep} / \mathrm{Et}$ & Gasper 1621 \\
\hline
\end{tabular}


Tabela 1. Continuação

\begin{tabular}{|c|c|c|c|c|c|}
\hline Família & Espécie & Distribuição & Fitofisionomia & Habitat & Voucher \\
\hline & Lastreopsis effusa (Sw.) Tindale & América Tropical & $\mathrm{D} / \mathrm{E}$ & Et & Verdi 1737 \\
\hline & Megalastrum abundans (Rosenst.) A.R. Sm. \& R.C. Moran & América do Sul & $\mathrm{D} / \mathrm{M}$ & Et & Dreveck 848 \\
\hline & Megalastrum connexum (Kaulf.) A.R. Sm. \& R.C. Moran & América do Sul & $\mathrm{D} / \mathrm{M} / \mathrm{E}$ & Et & Gasper 1669 \\
\hline & Megalastrum oreocharis (Sehnem) Salino \& Ponce & América do Sul & $\mathrm{D} / \mathrm{M} / \mathrm{E}$ & Et & Gasper 1614 \\
\hline & Megalastrum umbrinum (C.Chr.) A.R. Sm. \& R.C. Moran & América do Sul & $\mathrm{D}$ & Et & Korte 3884 \\
\hline & Mickelia guianensis (Aubl.) R.C. Moran et al. & América do Sul & $\mathrm{D}$ & Es & Schmitt 27 \\
\hline & Olfersia cervina (L.) Kunze & América Tropical & $\mathrm{D}$ & $\mathrm{Ep} / \mathrm{Et} / \mathrm{R}$ & Korte 2083 \\
\hline & Polybotrya cylindrica Kaulf. & Endêmica do Brasil & $\mathrm{D}$ & Es & Cadorin 60 \\
\hline & Polystichum montevidense (Spreng.) Rosenst. & América do Sul & $\mathrm{D} / \mathrm{M}$ & Et & Gasper 1789 \\
\hline & Polystichum pallidum Gardner & Endêmica do Brasil & M & Et & Gasper 1584 \\
\hline & Polystichum platyphyllum (Willd.) C. Presl & América Tropical & M & Et & Korte 7002 \\
\hline & Rumohra adiantiformis (G. Forst.) Ching & Pantropical & $\mathrm{D} / \mathrm{M} / \mathrm{R}$ & $\mathrm{Ep} / \mathrm{Et} / \mathrm{R}$ & Verdi 401 \\
\hline & Stigmatopteris brevinervis (Fée) R.C. Moran & Endêmica do Brasil & $\mathrm{D}$ & Et & Verdi 4693 \\
\hline & Stigmatopteris caudata (Raddi) C. Chr. & Endêmica do Brasil & $\mathrm{D}$ & Et & Schmitt 350 \\
\hline & Stigmatopteris heterocarpa (Fée) Rosenst. & Endêmica do Brasil & $\mathrm{D}$ & Et & Gasper 2296 \\
\hline Equisetaceae & Equisetum giganteum $\mathrm{L}$. & América Tropical & $\mathrm{D}$ & Et & Verdi 3172 \\
\hline \multirow[t]{7}{*}{ Gleicheniaceae } & Dicranopteris nervosa (Kaulf.) Maxon & América do Sul & $\mathrm{D} / \mathrm{M}$ & Et & Dreveck 123 \\
\hline & Gleichenella pectinata (Willd.) Ching & América Tropical & $\mathrm{D} / \mathrm{M}$ & Et & Gasper 2367 \\
\hline & Sticherus bifidus (Willd.) Ching & América Tropical & $\mathrm{D} / \mathrm{M}$ & Et & Dreveck 948 \\
\hline & Sticherus lanuginosus (Fée) Nakai & América do Sul & M & Et & Gasper 925 \\
\hline & Sticherus nigropaleaceus (J.W.Sturm) J. Prado \& Lellinger & América do Sul & $\mathrm{D}$ & Et & $\begin{array}{l}\text { Stival-Santos } \\
1741\end{array}$ \\
\hline & Sticherus pruinosus (Mart.) Ching & América do Sul & M & Et & Gasper 1423 \\
\hline & Sticherus squamosus (Fée) J. Gonzáles & Endêmica do Brasil & $\mathrm{D}$ & Et & Gasper 2140 \\
\hline \multirow[t]{19}{*}{ Hymenophyllaceae } & Abrodictyum rigidum (Sw.) Ebihara \& Dubuisson & Pantropical & $\mathrm{D}$ & $\mathrm{Ep} / \mathrm{Et} / \mathrm{R}$ & Gasper 2302 \\
\hline & Didymoglossum hymenoides (Hedw.) Desv. & América tropical & $\mathrm{D}$ & Ep & Schmitt 923 \\
\hline & Didymoglossum krausii (Hook. \& Grev.) C. Presl & América tropical & $\mathrm{D}$ & Ep & Schmitt 67 \\
\hline & Hymenophyllum asplenioides (Sw.) Sw. & América Tropical & $\mathrm{D} / \mathrm{M}$ & $\mathrm{Ep} / \mathrm{R}$ & Gasper 1657 \\
\hline & Hymenophyllum caudiculatum Mart. & América do Sul & $\mathrm{D} / \mathrm{M}$ & $\mathrm{Ep} / \mathrm{R}$ & Gasper 1516 \\
\hline & Hymenophyllum delicatulum Sehnem & Endêmica do sul do Brasil & $\mathrm{D}$ & Ep & Cadorin 1138 \\
\hline & Hymenophyllum fragile (Hedw.) C.V. Morton & América tropical & $\mathrm{D} / \mathrm{M}$ & $\mathrm{Ep} / \mathrm{R}$ & Schmitt 779 \\
\hline & Hymenophyllum fucoides (Sw.) Sw. & América tropical & $\mathrm{D} / \mathrm{M}$ & Ep & Gasper 1791 \\
\hline & Hymenophyllum hirsutum (L.) Sw. & América Tropical & $\mathrm{D}$ & Ep & Schmitt 37 \\
\hline & Hymenophyllum magellanicum Willd. ex Kunze & América do Sul & $\mathrm{D} / \mathrm{M}$ & Ep & Gasper 1882 \\
\hline & Hymenophyllum microcarpum Desv. & América tropical & $\mathrm{D}$ & $\mathrm{Ep} / \mathrm{R}$ & Cadorin 486 \\
\hline & Hymenophyllum polyanthos (Sw.) Sw. & Pantropical & $\mathrm{D} / \mathrm{M}$ & Ep & Gasper 1627 \\
\hline & Hymenophyllum rufum Fée & América Tropical & $\mathrm{D} / \mathrm{M}$ & Ep & Gasper 1658 \\
\hline & Hymenophyllum ulei Christ \& Giesenh. & Endêmica do sul do Brasil & $\mathrm{D}$ & Ep & Cadorin 2208 \\
\hline & $\begin{array}{l}\text { Polyphlebium angustatum (Carmich.) Ebihara \& } \\
\text { Dubuisson }\end{array}$ & América Tropical & $\mathrm{D} / \mathrm{M}$ & Ep & Gasper 1866 \\
\hline & Polyphlebium diaphanum (Kunth) Ebihara \& Dubuisson & América Tropical & $\mathrm{D}$ & $\mathrm{Ep} / \mathrm{R}$ & Cadorin 570 \\
\hline & Polyphlebium pyxidiferum (L.) Ebihara \& Dubuisson & América Tropical & $\mathrm{D}$ & Ep & Cadorin 62 \\
\hline & Trichomanes anadromum Rosenst. & América Tropical & $\mathrm{D} / \mathrm{M}$ & Ep & Gasper 1865 \\
\hline & Trichomanes cristatum Kaulf. & América do Sul & $\mathrm{D}$ & $\mathrm{Ep} / \mathrm{Et}$ & Gasper 2333 \\
\hline
\end{tabular}


Tabela 1. Continuação

\begin{tabular}{|c|c|c|c|c|c|}
\hline Família & Espécie & Distribuição & Fitofisionomia & Habitat & Voucher \\
\hline & Trichomanes elegans Rich. & América Tropical & $\mathrm{D}$ & Et & Korte 310 \\
\hline & Trichomanes pilosum Raddi & América do Sul & $\mathrm{D} / \mathrm{M}$ & $\mathrm{Ep} / \mathrm{Et}$ & Cadorin 466 \\
\hline & Trichomanes polypodioides $\mathrm{L}$. & América do Sul & $\mathrm{D} / \mathrm{M}$ & $\mathrm{Ep} / \mathrm{Et}$ & Gasper 1481 \\
\hline & Vandenboschia radicans (Sw.) Copel. & Pantropical & $\mathrm{D} / \mathrm{M} / \mathrm{E}$ & $\mathrm{Ep} / \mathrm{Es} / \mathrm{R}$ & Gasper 1497 \\
\hline & Vandenboschia rupestris (Raddi) Ebihara \& K. Iwats. & América Tropical & $\mathrm{D}$ & $\mathrm{Ep} / \mathrm{R}$ & Gasper 2308 \\
\hline Isoetaceae & Isoetes smithii H.P.Fuchs & Endêmica de Santa Catarina & M & $\mathrm{H}$ & Gasper 2963 \\
\hline \multirow[t]{9}{*}{ Lindsaeaceae } & Lindsaea bifida (Kaulf.) Mett. ex Kuhn & Endêmica do Brasil & $\mathrm{D}$ & Et & Stival-Santos 844 \\
\hline & Lindsaea botrychioides A. St.-Hil. & Endêmica do Sul e Sudeste & $\mathrm{D} / \mathrm{M}$ & $\mathrm{Ep} / \mathrm{Et}$ & Gasper 888 \\
\hline & Lindsaea lancea (L.) Bedd. & América Tropical & $\mathrm{D}$ & Et & Verdi 2492 \\
\hline & Lindsaea ovoidea Fée & Endêmica do Brasil & $\mathrm{D} / \mathrm{M}$ & $\mathrm{Ep} / \mathrm{Et}$ & Gasper 1556 \\
\hline & Lindsaea quadrangularis subsp. pallescens Sehnem & América do Sul & $\mathrm{D}$ & Et & Korte 333 \\
\hline & Lindsaea quadrangularis Raddi subsp. quadrangularis & Endêmica do Sul e Sudeste & $\mathrm{D}$ & $\mathrm{Ep} / \mathrm{Et}$ & Korte 940 \\
\hline & Lindsaea quadrangularis subsp. terminalis K.U. Kramer & Endêmica do sul do Brasil & $\mathrm{D}$ & Et & Verdi 3231 \\
\hline & Lindsaea virescens (Sw.) var. virescens & Endêmica do Sul e Sudeste & $\mathrm{D}$ & Et & Korte 978 \\
\hline & Lindsaea virescens var. catharinae (Hook.) Baker & Endêmica do Sul e Sudeste & $\mathrm{D}$ & Et & Cadorin 362 \\
\hline Lomariopsidaceae & Lomariopsis marginata (Schrad.) Kuhn & Endêmica do Brasil & $\mathrm{D}$ & $\mathrm{Et} / \mathrm{Es}$ & Gasper 2237 \\
\hline \multirow[t]{3}{*}{ Lomariopsidaceae } & Nephrolepis cordifolia (L.) C. Presl & Introduzida & $\mathrm{D}$ & Ep & Schmitt 86 \\
\hline & Nephrolepis pectinata (Willd.) Schott & América Tropical & $\mathrm{D} / \mathrm{E}$ & $\mathrm{Ep} / \mathrm{Et} / \mathrm{R}$ & Gasper 1969 \\
\hline & Nephrolepis pendula (Raddi) J. Sm. & América Tropical & $\mathrm{D}$ & $\mathrm{Ep} / \mathrm{Et} / \mathrm{R}$ & Cadorin 48 \\
\hline \multirow[t]{16}{*}{ Lycopodiaceae } & Huperzia acerosa (Sw.) Holub & América Tropical & $\mathrm{D}$ & Ep & Cadorin 746 \\
\hline & Huperzia biformis (Hook.) Holub & Endêmica do Sul e Sudeste & $\mathrm{D}$ & Ep & Korte 4156 \\
\hline & $\begin{array}{l}\text { Huperzia comans (Herter ex Nessel) B. Øllg. \& P.G. } \\
\text { Windisch }\end{array}$ & Endêmica do Sul e Sudeste & $\mathrm{D}$ & Ep & Cadorin 2893 \\
\hline & Huperzia flexibilis (Fée) B. Øllg. & Endêmica do Brasil & $\mathrm{D}$ & Ep & Cadorin 53 \\
\hline & Huperzia fontinaloides (Spring) Trevis. & Endêmica do Sul e Sudeste & $\mathrm{D} / \mathrm{M}$ & Ep & Gasper 1654 \\
\hline & Huperzia heterocarpon (Fée) Holub & América do Sul & $\mathrm{D} / \mathrm{M}$ & Ep & Gasper 1249 \\
\hline & Huperzia hexasticha B. Øllg. \& P.G. Windisch & Endêmica do Sul e Sudeste & $\mathrm{D} / \mathrm{M}$ & Ep & Schmitt 21 \\
\hline & Huperzia mandiocana (Raddi) Trevis. & América do Sul & $\mathrm{D} / \mathrm{M}$ & Ep & Gasper 1830 \\
\hline & Huperzia mollicoma (Spring) Holub & América Tropical & $\mathrm{D}$ & Ep & Schmitt 1666 \\
\hline & Huperzia quadrifariata (Bory) Rothm. & Endêmica do Brasil & $\mathrm{D}$ & Ep & Schmitt 1063 \\
\hline & Huperzia reflexa (Lam.) Trevis. & América Tropical & $\mathrm{D} / \mathrm{M}$ & $\mathrm{Et} / \mathrm{R}$ & Gasper 1656 \\
\hline & Lycopodiella alopecuroides (L.) Cranfill & América Tropical & $\mathrm{D} / \mathrm{M}$ & Et & Gasper 129 \\
\hline & Lycopodiella caroliniana (L.) Pic. Serm. & Pantropical & $\mathrm{D} / \mathrm{M}$ & Et & Gasper 1756 \\
\hline & Lycopodiella cernua (L.) Pic. Serm. & Pantropical & $\mathrm{D} / \mathrm{M}$ & $\mathrm{Et} / \mathrm{R}$ & Gasper 1665 \\
\hline & Lycopodium clavatum $\mathrm{L}$. & Pantropical & $\mathrm{D} / \mathrm{M}$ & Et & Gasper 2449 \\
\hline & Lycopodium thyoides Humb. \& Bonpl. ex Willd. & América Tropical & $\mathrm{D} / \mathrm{M}$ & $\mathrm{Et} / \mathrm{R}$ & Gasper 1660 \\
\hline Lygodiaceae & Lygodium volubile Sw. & Pantropical & $\mathrm{D} / \mathrm{R}$ & Es & Gasper 2232 \\
\hline \multirow[t]{5}{*}{ Marattiaceae } & Danaea geniculata Raddi & América Tropical & $\mathrm{D}$ & $\mathrm{Et} / \mathrm{R}$ & Verdi 3343 \\
\hline & Danaea moritziana C. Presl & América Tropical & $\mathrm{D}$ & Et & Gasper 2213 \\
\hline & Danaea sellowiana C. Presl & América Tropical & $\mathrm{D}$ & Et & Cadorin 2169 \\
\hline & Eupodium kaulfussii (J.Sm.) J.Sm. & América do Sul & $\mathrm{D} / \mathrm{M}$ & $\mathrm{Et} / \mathrm{R}$ & Gasper 1710 \\
\hline & Marattia cicutifolia Kaulf. & Endêmica do Brasil & $\mathrm{D} / \mathrm{M}$ & Et & Gasper 1558 \\
\hline \multirow[t]{3}{*}{ Ophioglossaceae } & Botrychium virginianum (L.) Sw. & Cosmopolita (exceto África) & $\mathrm{E}$ & Et & Gasper 1960 \\
\hline & Ophioglossum palmatum L. & Pantropical & $\mathrm{D}$ & $\mathrm{Ep} / \mathrm{R}$ & Cadorin 481 \\
\hline & Ophioglossum reticulatum $\mathrm{L}$. & Pantropical & M & Et & Gasper 1463 \\
\hline
\end{tabular}


Tabela 1. Continuação

\begin{tabular}{|c|c|c|c|c|c|}
\hline Família & Espécie & Distribuição & Fitofisionomia & Habitat & Voucher \\
\hline \multirow[t]{2}{*}{ Osmundaceae } & Osmunda regalis $\mathrm{L}$. & Pantropical & M & Et & Gasper 1611 \\
\hline & Osmundastrum cinnamomeum (L.) C. Presl & Pantropical & $\mathrm{D} / \mathrm{R}$ & Et & Gasper 2470 \\
\hline Plagyogiriaceae & Plagiogyria fialhoi (Fée \& Glaz.) Copel. & Endêmica do Sul e Sudeste & M & Et & Gasper 2980 \\
\hline \multirow[t]{39}{*}{ Polypodiaceae } & Alansmia reclinata (Brack.) Moguel \& M. Kessler & Endêmica do Sul e Sudeste & $\mathrm{D} / \mathrm{M}$ & Ep & Verdi 673 \\
\hline & Alansmia senilis (Fée) Moguel \& M.Kessler & América do Sul & M & $\mathrm{R}$ & Gasper 2955 \\
\hline & Campyloneurum acrocarpon Fée & Endêmica do Brasil & $\mathrm{D} / \mathrm{M} / \mathrm{E}$ & $\mathrm{Ep} / \mathrm{Et} / \mathrm{R}$ & Cadorin 97 \\
\hline & Campyloneurum austrobrasilianum (Alston) de la Sota & Endêmica do Brasil & $\mathrm{D} / \mathrm{M}$ & $\mathrm{Ep} / \mathrm{Et}$ & Gasper 1048 \\
\hline & Campyloneurum decurrens (Raddi) C. Presl & Endêmica do Brasil & $\mathrm{D}$ & $\mathrm{Et} / \mathrm{R}$ & Cadorin 628 \\
\hline & Campyloneurum minus Fée & América do Sul & $\mathrm{D} / \mathrm{M} / \mathrm{E}$ & $\mathrm{Ep} / \mathrm{Et} / \mathrm{R}$ & Gasper 1209 \\
\hline & Campyloneurum nitidum (Kaulf.) C. Presl & América do Sul & $\mathrm{D} / \mathrm{M} / \mathrm{E}$ & $\mathrm{Ep} / \mathrm{Et} / \mathrm{R}$ & Verdi 129 \\
\hline & Campyloneurum rigidum J. Sm. & América do Sul & $\mathrm{D} / \mathrm{M}$ & $\mathrm{Ep} / \mathrm{Et} / \mathrm{R}$ & Gasper 1111 \\
\hline & Ceradenia albidula (Baker) L.E. Bishop & Endêmica do Sul e Sudeste & $\mathrm{D} / \mathrm{M}$ & Ep & Gasper 1116 \\
\hline & Ceradenia spixiana (Mart. ex Mett.) L.E. Bishop & América tropical & $\mathrm{D}$ & Ep & Cadorin 743 \\
\hline & Cochlidium punctatum (Raddi) L.E. Bishop & Endêmica do Brasil & $\mathrm{D}$ & Ep & Cadorin 484 \\
\hline & Cochlidium serrulatum (Sw.) L.E. Bishop & Pantropical & $\mathrm{D} / \mathrm{M}$ & $\mathrm{Ep} / \mathrm{R}$ & Cadorin 63 \\
\hline & $\begin{array}{l}\text { Lellingeria brevistipes (Mett. ex Kuhn) A.R. Sm. \& R.C. } \\
\text { Moran }\end{array}$ & Endêmica do Sul e Sudeste & $\mathrm{D} / \mathrm{M}$ & $\mathrm{Ep} / \mathrm{R}$ & Schmitt 1114 \\
\hline & Lellingeria depressa (C.Chr.) A.R. Sm. \& R.C. Moran & Endêmica do Sul e Sudeste & $\mathrm{D}$ & Ep & Cadorin 2566 \\
\hline & Leucotrichum organense (Gardner) Labiak & Endêmica do Brasil & $\mathrm{D} / \mathrm{M}$ & Ep & Verdi 2023 \\
\hline & Leucotrichum schenckii (Hieron.) Labiak & Endêmica do Sul e Sudeste & $\mathrm{D} / \mathrm{M}$ & Ep & Gasper 1618 \\
\hline & Melpomene flabelliformis (Poir.) A.R. Sm. \& R.C. Moran & Anfiatlântico & $\mathrm{D}$ & $\mathrm{Ep} / \mathrm{R}$ & Verdi 2113 \\
\hline & $\begin{array}{l}\text { Melpomene pilosissima (M. Martens \& Galeotti) A.R. Sm. } \\
\text { \& R.C. Moran }\end{array}$ & América Tropical & $\mathrm{D}$ & Ep & Cadorin 3014 \\
\hline & Microgramma percussa (Cav.) de la Sota & América tropical & $\mathrm{D}$ & Ep & Stival-Santos 817 \\
\hline & Microgramma squamulosa (Kaulf.) de la Sota & América do Sul & $\mathrm{D} / \mathrm{M} / \mathrm{E} / \mathrm{R}$ & Ep & Verdi 285 \\
\hline & Microgramma tecta (Kaulf.) Alston. & América tropical & $\mathrm{D}$ & Ep & Gasper 2199 \\
\hline & Microgramma vacciniifolia (Langsd. \& Fisch.) Copel. & América do Sul & $\mathrm{D} / \mathrm{M} / \mathrm{R}$ & Ep & Gasper 1011 \\
\hline & Moranopteris achilleifolia (Kaulf.) R.Y. Hirai \& J. Prado & Endêmica do Brasil & $\mathrm{D} / \mathrm{M}$ & Ep & Gasper 1659 \\
\hline & Niphidium crassifolium (L.) Lellinger & América Tropical & $\mathrm{D} / \mathrm{M} / \mathrm{E}$ & $\mathrm{Ep} / \mathrm{Et} / \mathrm{R}$ & Dreveck 158 \\
\hline & Pecluma chnoophora (Kunze) Salino \& F.C. Assis & Endêmica do Brasil & $\mathrm{D}$ & $\mathrm{Ep} / \mathrm{Et}$ & Gasper 2340 \\
\hline & Pecluma paradiseae (Langsd. \& Fisch.) M.G. Price & Endêmica do Brasil & $\mathrm{D} / \mathrm{M} / \mathrm{R}$ & $\mathrm{Ep} / \mathrm{Et}$ & Verdi 482 \\
\hline & Pecluma pectinatiformis (Lindm.) M.G. Price & América do Sul & $\mathrm{D} / \mathrm{M}$ & $\mathrm{Ep} / \mathrm{Et}$ & Gasper 1594 \\
\hline & Pecluma recurvata (Kaulf.) M.G. Price & América do Sul & $\mathrm{D} / \mathrm{M}$ & $\begin{array}{l}\mathrm{Ep} / \mathrm{Et} / \\
\mathrm{Es} / \mathrm{R}\end{array}$ & Gasper 911 \\
\hline & Pecluma robusta (Fée) M. Kessler \& A.R. Sm. & América Tropical & $\mathrm{D} / \mathrm{E}$ & $\mathrm{Ep} / \mathrm{Et}$ & Gasper 2364 \\
\hline & Pecluma sicca (Lindm.) M.G. Price & América do Sul & $\mathrm{D} / \mathrm{M} / \mathrm{E}$ & $\mathrm{Ep} / \mathrm{Et} / \mathrm{R}$ & Gasper 1720 \\
\hline & Pecluma singeri (de la Sota) M.G. Price & América do Sul & $\mathrm{D} / \mathrm{M} / \mathrm{E}$ & $\mathrm{Ep} / \mathrm{Et} / \mathrm{R}$ & Gasper 1309 \\
\hline & Pecluma truncorum (Lindm.) M.G. Price & América do Sul & $\mathrm{D} / \mathrm{M}$ & Ep & Gasper 1555 \\
\hline & Phlebodium pseudoaureum (Cav.) Lellinger & América Tropical & $\mathrm{D}$ & Ep & Cadorin 806 \\
\hline & Pleopeltis astrolepis (Liebm.) E. Fourn. & América Tropical & $\mathrm{D} / \mathrm{R}$ & Ep & Gasper 2247 \\
\hline & Pleopeltis hirsutissima (Raddi) de la Sota & América do Sul & $\mathrm{D} / \mathrm{M} / \mathrm{E} / \mathrm{R}$ & $\mathrm{Ep} / \mathrm{R}$ & Gasper 1870 \\
\hline & Pleopeltis lepidopteris (Langsd. \& Fisch.) de la Sota & América do Sul & $\mathrm{R}$ & $\mathrm{Et} / \mathrm{R}$ & $\begin{array}{l}\text { Stival-Santos } \\
1918\end{array}$ \\
\hline & Pleopeltis macrocarpa (Bory ex Willd.) Kaulf. & Pantropical & $\mathrm{D} / \mathrm{M}$ & Ep & Gasper 2009 \\
\hline & Pleopeltis minima (Bory) J. Prado \& R.Y. Hirai & América do Sul & $\mathrm{D} / \mathrm{M} / \mathrm{E}$ & $\mathrm{Ep} / \mathrm{Et} / \mathrm{R}$ & Dreveck 489 \\
\hline & Pleopeltis pleopeltidis (Fée) de la Sota & América do Sul & $\mathrm{D} / \mathrm{M}$ & Ep & Gasper 1310 \\
\hline
\end{tabular}


André Luís de Gasper, Alexandre Salino, Alexander C. Vibrans, Lucia Sevegnani, Marcio Verdi, Alexandre Korte, Anita Stival dos Santos, Susana Dreveck, Tiago João Cadorin, Juliane Luzia Schmitt e Eder Caglioni

Tabela 1. Continuação

\begin{tabular}{|c|c|c|c|c|c|}
\hline Família & Espécie & Distribuição & Fitofisionomia & Habitat & Voucher \\
\hline & Pleopeltis pleopeltifolia (Raddi) Alston & América do Sul & $\mathrm{D} / \mathrm{M} / \mathrm{E} / \mathrm{R}$ & $\mathrm{Ep} / \mathrm{Et} / \mathrm{R}$ & Gasper 1988 \\
\hline & Serpocaulon catharinae (Langsd. \& Fisch.) A.R. Sm. & América do Sul & $\mathrm{D} / \mathrm{M}$ & $\mathrm{Ep} / \mathrm{Et} / \mathrm{R}$ & Gasper 1138 \\
\hline & Serpocaulon fraxinifolium (Jacq.) A.R. Sm. & América Tropical & $\mathrm{D}$ & Ep/Es & Verdi 2497 \\
\hline & Serpocaulon latipes (Langsd. \& Fisch.) A.R. Sm. & América Tropical & $\mathrm{D} / \mathrm{M}$ & $\mathrm{Ep} / \mathrm{Et} / \mathrm{R}$ & Cadorin 82 \\
\hline & Serpocaulon meniscifolium (Langsd. \& Fisch.) A.R. Sm. & Endêmica do Brasil & $\mathrm{D}$ & Ep & Cadorin 1541 \\
\hline & Serpocaulon triseriale (Sw.) A.R. Sm. & América tropical & $\mathrm{D}$ & $\mathrm{Et} / \mathrm{R}$ & Dreveck 1903 \\
\hline & Serpocaulon vacillans (Link) A.R. Sm. & América do Sul & $\mathrm{D} / \mathrm{R}$ & $\mathrm{Ep} / \mathrm{Et}$ & Schmitt 32 \\
\hline & Terpsichore chrysleri (Proctor ex Copel.) A.R. Sm. & América do Sul & $\mathrm{D}$ & Ep & Gasper 2214 \\
\hline & Zygophlebia longipilosa (C. Chr.) L.E. Bishop & Endêmica do Sul e Sudeste & $\mathrm{D}$ & Ep & Cadorin 2909 \\
\hline Psilotaceae & Psilotum nudum (L.) P. Beauv. & $\begin{array}{l}\text { América tropical e } \\
\text { Paleotrópicos }\end{array}$ & $\mathrm{D}$ & $\mathrm{Ep} / \mathrm{Et}$ & Cadorin 1711 \\
\hline \multirow[t]{32}{*}{ Pteridaceae } & Acrostichum danaeifolium Langsd. \& Fisch. & $\begin{array}{l}\text { América tropical e } \\
\text { Paleotrópicos }\end{array}$ & $\mathrm{D}$ & Et & Cadorin 1622 \\
\hline & Adiantopsis chlorophylla (Sw.) Fée & América do Sul & $\mathrm{D} / \mathrm{M} / \mathrm{E}$ & Et & Gasper 895 \\
\hline & Adiantopsis perfasciculata Sehnem & Endêmica do Brasil & $\mathrm{M} / \mathrm{E}$ & Et & Korte 6659 \\
\hline & Adiantopsis radiata (L.) Fée & América tropical & $\mathrm{D}$ & $\mathrm{Et} / \mathrm{R}$ & Verdi 2592 \\
\hline & Adiantopsis regularis (Mett.) T. Moore & América do Sul & $\mathrm{D} / \mathrm{M}$ & Et & Korte 1995 \\
\hline & Adiantum abscissum Schrad. & Endêmica do Brasil & $\mathrm{D}$ & Et & Dreveck 2317 \\
\hline & Adiantum curvatum Kaulf. & América do Sul & $\mathrm{D}$ & Et & Korte 317 \\
\hline & Adiantum pentadactylon Langsd. \& Fisch. & Endêmica do Brasil & $\mathrm{D}$ & Et & $\begin{array}{l}\text { Stival-Santos } \\
1430\end{array}$ \\
\hline & Adiantum pseudotinctum Hieron. & América do Sul & $\mathrm{D} / \mathrm{M} / \mathrm{E}$ & $\mathrm{Et} / \mathrm{R}$ & Gasper 1344 \\
\hline & Adiantum raddianum C. Presl & América Tropical & $\mathrm{D} / \mathrm{M} / \mathrm{E}$ & Et & Gasper 994 \\
\hline & Anogramma chaerophylla (Desv.) Link & América do Sul & $\mathrm{E}$ & Et & Gasper 1955 \\
\hline & Cheilanthes micropteris Sw. & América do Sul & M & $\mathrm{R}$ & Korte 7014 \\
\hline & Doryopteris acutiloba (Prantl) Diels & América do Sul & M & Et & Verdi 168 \\
\hline & Doryopteris collina (Raddi) J. Sm. & América do Sul & $\mathrm{D} / \mathrm{M} / \mathrm{E}$ & $\mathrm{Et} / \mathrm{R}$ & Gasper 1313 \\
\hline & Doryopteris concolor (Langsd. \& Fisch.) Kuhn & Pantropical & $\mathrm{D} / \mathrm{M} / \mathrm{E}$ & Et & Gasper 1961 \\
\hline & Doryopteris crenulans (Fée) Christ & América do Sul & $\mathrm{D} / \mathrm{M}$ & Et & Dreveck 121 \\
\hline & Doryopteris lomariacea Klotzsch & América tropical & M & Et & Korte 1104 \\
\hline & Doryopteris lorentzii (Hieron.) Diels & América do Sul & $\mathrm{D} / \mathrm{M}$ & $\mathrm{Ep} / \mathrm{Et}$ & Verdi 556 \\
\hline & Doryopteris majestosa Yesilyurt & América do Sul & $\mathrm{D} / \mathrm{M} / \mathrm{E}$ & Et & Gasper 1312 \\
\hline & Doryopteris nobilis (T. Moore) C. Chr. & Endêmica do Brasil & $\mathrm{D}$ & $\mathrm{R}$ & Verdi 4531 \\
\hline & Doryopteris pentagona Pic. Serm. & América do Sul & $\mathrm{D} / \mathrm{M} / \mathrm{E}$ & $\mathrm{Et} / \mathrm{R}$ & Gasper 1681 \\
\hline & Doryopteris raddiana Fée & Endêmica do Brasil & $\mathrm{D}$ & $\mathrm{Et} / \mathrm{R}$ & Cadorin 2733 \\
\hline & Doryopteris sagittifolia (Raddi) J. Sm. & América do Sul & $\mathrm{D}$ & $\mathrm{R}$ & Cadorin 554 \\
\hline & Doryopteris triphylla (Lam.) Christ & América do Sul & $\mathrm{D}$ & $\mathrm{Et} / \mathrm{R}$ & Gasper 1805 \\
\hline & Doryopteris varians $\mathrm{Sm}$. & América do Sul & $\mathrm{D}$ & Et & $\begin{array}{l}\text { Stival-Santos } \\
1173\end{array}$ \\
\hline & Hemionitis tomentosa (Lam.) Raddi & América do Sul & $\mathrm{D} / \mathrm{E}$ & $\mathrm{Et} / \mathrm{R}$ & Verdi 1582 \\
\hline & Jamesonia myriophylla (Sw.) Christenh. & América do Sul & M & Et & Gasper 1662 \\
\hline & Pityrogramma calomelanos (L.) Link & América Tropical & $\mathrm{D}$ & $\mathrm{Et} / \mathrm{R}$ & Gasper 2144 \\
\hline & Polytaenium cajanense (Desv.) Benedict & América Tropical & $\mathrm{D}$ & Ep & Cadorin 183 \\
\hline & Pteris altissima Poir. & América Tropical & $\mathrm{D}$ & Et & Gasper 2313 \\
\hline & Pteris angustata (Fée) C.V. Morton & Endêmica do Sul e Sudeste & $\mathrm{D}$ & Et & Verdi 3346 \\
\hline & Pteris brasiliense Raddi & América do Sul & $\mathrm{D}$ & Et & Cadorin 1727 \\
\hline
\end{tabular}


Tabela 1. Continuação

\begin{tabular}{|c|c|c|c|c|c|}
\hline Família & Espécie & Distribuição & Fitofisionomia & Habitat & Voucher \\
\hline & Pteris decurrens C. Presl & América Tropical & $\mathrm{D} / \mathrm{M}$ & Et & Gasper 992 \\
\hline & Pteris deflexa Link & América Tropical & $\mathrm{D} / \mathrm{M} / \mathrm{E}$ & Et & Gasper 1372 \\
\hline & Pteris denticulata var. tristicula (Raddi) J. Prado & América do Sul & $\mathrm{D}$ & Et & Verdi 3033 \\
\hline & Pteris denticulata Sw. var. denticulata & América tropical & $\mathrm{D} / \mathrm{M} / \mathrm{E}$ & Et & Verdi 4335 \\
\hline & Pteris lechleri Mett. & América Tropical & $\mathrm{D} / \mathrm{M} / \mathrm{E}$ & Et & Gasper 1814 \\
\hline & Pteris longifolia $\mathrm{L}$. & Introduzida & $\mathrm{D}$ & $\mathrm{Ep} / \mathrm{Et} / \mathrm{R}$ & Schmitt 391 \\
\hline & Pteris splendens Kaulf. & América do Sul & $\mathrm{D} / \mathrm{E}$ & $\mathrm{Et} / \mathrm{R}$ & Gasper 1685 \\
\hline & Radiovittaria stipitata (Kunze) E.H. Crane & América Tropical & $\mathrm{D}$ & Ep & Gasper 2220 \\
\hline & Vittaria lineata (L.) Sm. & América Tropical & $\mathrm{D} / \mathrm{M}$ & $\mathrm{Ep} / \mathrm{R}$ & Gasper 1642 \\
\hline & Vittaria scabrida Klotzsch & América do Sul & $\mathrm{D} / \mathrm{M} / \mathrm{R}$ & $\mathrm{Ep} / \mathrm{R}$ & Gasper 893 \\
\hline \multirow[t]{2}{*}{ Saccolomataceae } & Saccoloma elegans Kaulf. & América Tropical & $\mathrm{D}$ & Et & Gasper 2225 \\
\hline & Saccoloma inaequale (Kunze) Mett. & América Tropical & $\mathrm{D}$ & Et & Korte 215 \\
\hline \multirow[t]{2}{*}{ Salviniaceae } & Azolla caroliniana Willd. & América Tropical & M & $\mathrm{H}$ & Gasper 3002 \\
\hline & Salvinia auriculata Aubl. & América Tropical & $\mathrm{D}$ & $\mathrm{H}$ & Korte 6807 \\
\hline Schizaeaceae & Schizaea elegans (Vahl) Sw. & América Tropical & $\mathrm{D}$ & Et & Gasper 2366 \\
\hline \multirow[t]{5}{*}{ Selaginellaceae } & Selaginella contigua Baker & Endêmica do Brasil & $\mathrm{D}$ & Et & Gasper 2446 \\
\hline & Selaginella decomposita Spring & Endêmica do Brasil & $\mathrm{D}$ & Et & Korte 838 \\
\hline & Selaginella flexuosa Spring & América do Sul & $\mathrm{D}$ & $\mathrm{Ep} / \mathrm{Et} / \mathrm{R}$ & Gasper 2226 \\
\hline & Selaginella macrostachya (Spring) Spring & América do Sul & $\mathrm{D}$ & $\mathrm{Ep} / \mathrm{Et}$ & Cadorin 520 \\
\hline & Selaginella marginata (Humb \& Bonpl. ex Willd.) Spring & América Tropical & $\mathrm{D}$ & Et & Verdi 2154 \\
\hline \multirow[t]{2}{*}{ Selaginellaceae } & Selaginella muscosa Spring & América do Sul & $\mathrm{D} / \mathrm{M}$ & $\mathrm{Ep} / \mathrm{Et} / \mathrm{R}$ & Cadorin 350 \\
\hline & Selaginella sulcata (Desv.) Spring ex Mart. & América do Sul & $\mathrm{D} / \mathrm{M}$ & Et & Gasper 1664 \\
\hline \multirow[t]{4}{*}{ Tectariaceae } & Tectaria buchtienii (Rosenst.) Maxon & Endêmica do sul do Brasil & $\mathrm{D}$ & $\mathrm{Et} / \mathrm{R}$ & Gasper 2293 \\
\hline & Tectaria incisa Cav. & América Tropical & $\mathrm{D}$ & $\mathrm{Et} / \mathrm{R}$ & Gasper 2248 \\
\hline & Tectaria pilosa (Fée) R.C. Moran & América Tropical & $\mathrm{D}$ & Et & Verdi 2619 \\
\hline & Tectaria vivipara Jermy \& T.G. Walker & América Tropical & $\mathrm{D}$ & Et & Dreveck 1706 \\
\hline \multirow[t]{17}{*}{ Thelypteridaceae } & Macrothelypteris torresiana (Gaudich.) Ching & Introduzida & $\mathrm{D} / \mathrm{M} / \mathrm{E}$ & Et & Dreveck 457 \\
\hline & Thelypteris abbiattii C.F. Reed & América do Sul & $\mathrm{D}$ & Et & $\begin{array}{l}\text { Stival-Santos } \\
1724\end{array}$ \\
\hline & Thelypteris amambayensis Ponce & América do Sul & $\mathrm{D} / \mathrm{M}$ & Et & Gasper 1793 \\
\hline & Thelypteris burkartii Abbiatti & América do Sul & $\mathrm{D} / \mathrm{M}$ & Et & Dreveck 1446 \\
\hline & Thelypteris conspersa (Schrad.) A.R. Sm. & América tropical & $\mathrm{D} / \mathrm{M}$ & Et & Korte 3004 \\
\hline & Thelypteris decurtata (Link) de la Sota & América do Sul & M & Et & Gasper 331 \\
\hline & Thelypteris decussata var. brasiliensis (C.Chr.) A.R. Sm. & América do Sul & $\mathrm{D}$ & Et & Gasper 2428 \\
\hline & Thelypteris dentata (Forssk.) E.P. St. John & Introduzida & $\mathrm{D} / \mathrm{M} / \mathrm{E}$ & Et & Gasper 2057 \\
\hline & Thelypteris gymnosora Ponce & Endêmica do Brasil & $\mathrm{D} / \mathrm{M}$ & $\mathrm{Ep} / \mathrm{Et} / \mathrm{R}$ & Schmitt 924 \\
\hline & Thelypteris hatschbachii A.R. Sm. & Endêmica do Sul e Sudeste & $\mathrm{D} / \mathrm{M}$ & Et & Korte 1600 \\
\hline & Thelypteris hispidula (Decne.) C.F. Reed & Cosmopolita & $\mathrm{D} / \mathrm{M} / \mathrm{E}$ & Et & Verdi 470 \\
\hline & Thelypteris iguapensis (C. Chr.) Salino & Endêmica do Sul e Sudeste & $\mathrm{D}$ & Et & Korte 3309 \\
\hline & Thelypteris interrupta (Willd.) K. Iwats. & Cosmopolita & $\mathrm{D}$ & Et & Korte 4100 \\
\hline & Thelypteris juergensii (Rosenst.) C.F.Reed & Endêmica do sul do Brasil & M & Et & Gasper 2942 \\
\hline & Thelypteris longifolia (Desv.) R.M. Tryon & América tropical & $\mathrm{D}$ & Et & $\begin{array}{l}\text { Stival-Santos } \\
1126\end{array}$ \\
\hline & Thelypteris maxoniana A.R. Sm. & América tropical & $\mathrm{D}$ & $\mathrm{Et} / \mathrm{R}$ & Gasper 2429 \\
\hline & Thelypteris metteniana Ching & América do Sul & $\mathrm{D}$ & $\mathrm{Et} / \mathrm{R}$ & Gasper 2087 \\
\hline
\end{tabular}


André Luís de Gasper, Alexandre Salino, Alexander C. Vibrans, Lucia Sevegnani, Marcio Verdi, Alexandre Korte, Anita Stival dos Santos, Susana Dreveck, Tiago João Cadorin, Juliane Luzia Schmitt e Eder Caglioni

Tabela 1. Continuação

\begin{tabular}{|c|c|c|c|c|c|}
\hline Família & Espécie & Distribuição & Fitofisionomia & Habitat & Voucher \\
\hline \multirow[t]{11}{*}{ Thelypteridaceae } & Thelypteris opposita (Vahl) Ching & América Tropical & $\mathrm{D} / \mathrm{M}$ & Et & Stival-Santos 1664 \\
\hline & Thelypteris patens (Sw.) Small & América Tropical & $\mathrm{D}$ & Et & Stival-Santos 2200 \\
\hline & Thelypteris ptarmica (Mett.) C.F. Reed & Endêmica do Brasil & $\mathrm{D}$ & $\mathrm{Et} / \mathrm{R}$ & Gasper 2223 \\
\hline & Thelypteris raddii (Rosenst.) Ponce & Endêmica do Brasil & $\mathrm{D}$ & Et & Schmitt 617 \\
\hline & Thelypteris recumbens (Rosenst.) C.F. Reed & Endêmica do sul do Brasil & M & Et & Gasper 2011 \\
\hline & Thelypteris retusa (Sw.) C.F. Reed & Endêmica do Brasil & $\mathrm{D} / \mathrm{M}$ & Et & Stival-Santos 2502 \\
\hline & Thelypteris riograndensis (Lindm.) C.F. Reed & América do Sul & $\mathrm{D} / \mathrm{M}$ & Et & Gasper 1348 \\
\hline & Thelypteris sanctae-catharinae (Rosenst.) Ponce & Endêmica do sul do Brasil & M & Et & Korte 6910 \\
\hline & Thelypteris scabra (C. Presl) Lellinger & América do Sul & $\mathrm{D} / \mathrm{E}$ & Et & Gasper 1689 \\
\hline & Thelypteris serrata (Cav.) Alston & América tropical & $\mathrm{D}$ & Et & Korte 5011 \\
\hline & Thelypteris vivipara (Raddi) C.F. Reed & Endêmica do Brasil & $\mathrm{D}$ & Et & Korte 158 \\
\hline \multirow[t]{14}{*}{ Woodsiaceae } & Athyrium filix-femina (L.) Roth & Cosmopolita & M & Et & Gasper 2097 \\
\hline & Cystopteris fragilis (L.) Bernh. & Cosmopolita & M & Et & Gasper 2900 \\
\hline & Deparia petersenii (Kunze) M. Kato & Introduzida & $\mathrm{D} / \mathrm{M}$ & $\mathrm{Et} / \mathrm{R}$ & Dreveck 114 \\
\hline & Diplazium ambiguum Raddi & América do Sul & $\mathrm{D} / \mathrm{M}$ & Et & Gasper 2341 \\
\hline & Diplazium cristatum (Desr.) Alston & América tropical & $\mathrm{D} / \mathrm{M} / \mathrm{E}$ & $\mathrm{Ep} / \mathrm{Et} / \mathrm{R}$ & Gasper 1684 \\
\hline & Diplazium herbaceum Fée & Endêmica do sul do Brasil & $\mathrm{D} / \mathrm{M} / \mathrm{E}$ & Et & Gasper 1572 \\
\hline & Diplazium leptocarpon Fée & Endêmica do Brasil & $\mathrm{D} / \mathrm{M}$ & Et & Gasper 991 \\
\hline & Diplazium lindbergii (Mett.) Christ & América Tropical & $\mathrm{D}$ & Et & Gasper 2147 \\
\hline & Diplazium plantaginifolium (L.) Urb. & América Tropical & $\mathrm{D}$ & $\mathrm{Et} / \mathrm{R}$ & Gasper 2125 \\
\hline & Diplazium riedelianum (Bong. ex Kuhn) C. Chr. & América Tropical & $\mathrm{D}$ & $\mathrm{Ep} / \mathrm{Et}$ & Gasper 2128 \\
\hline & Diplazium rostratum Fée & América do Sul & $\mathrm{D} / \mathrm{M}$ & Et & Dreveck 933 \\
\hline & Diplazium striatum (L.) C. Presl & América Tropical & M & Et & Gasper 1946 \\
\hline & Diplazium turgidum Rosenst. & Endêmica do Brasil & $\mathrm{D} / \mathrm{M}$ & Et & Gasper 1029 \\
\hline & Hemidictyum marginatum (L.) C. Presl & América Tropical & $\mathrm{D}$ & Et & Stival-Santos 1063 \\
\hline
\end{tabular}

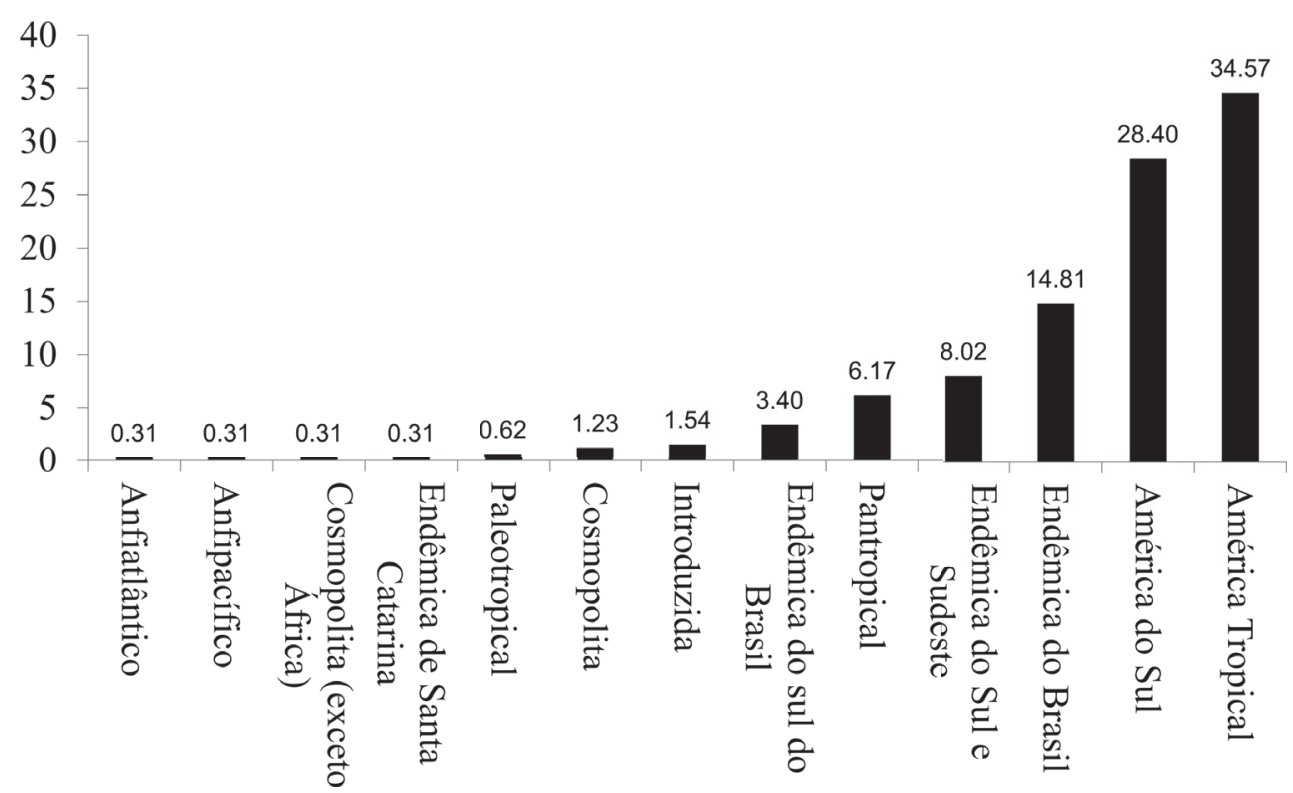

Figura 3. Distribuição geográfica das espécies de pteridófitas registradas em Santa Catarina. Valores expressos em porcentagem. 


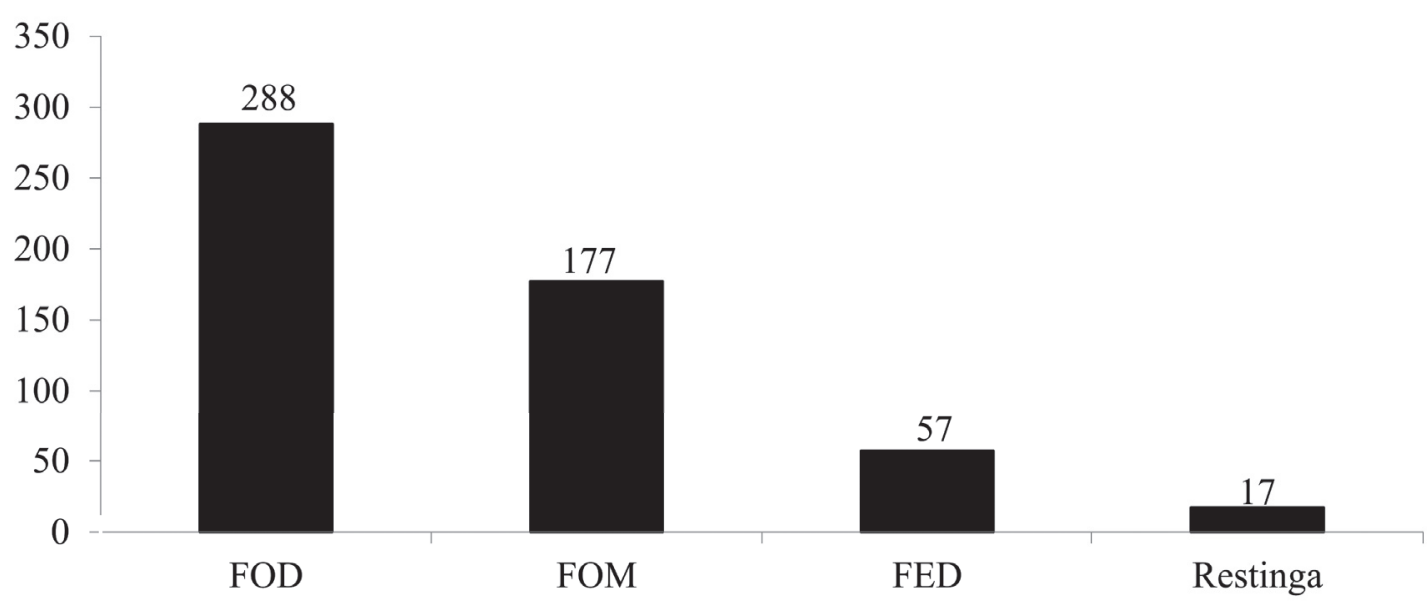

Figura 4. Distribuição das espécies de pteridófitas entre as regiões fitoecológicas e formações associadas de Santa Catarina. FOD: Floresta Ombrófila Densa; FOM: Floresta Ombrófila Mista, FED: Floresta Estacional Decidual.

Mesmo a Floresta Ombrófila Densa, que em uma única unidade de conservação (Parque Nacional da Serra do Itajaí) protege quase $50 \%$ das espécies de pteridófitas de Santa Catarina (Gasper \& Sevegnani 2010) carece de maior proteção, já que outras formações não cobertas pelo Parque como restinga, mangue, banhados e lagoas do sul do estado estão desprotegidas.

Mesmo com todos os trabalhos já realizados e tendo em vista o atual estado de fragmentação dos remanescentes no estado, novas espécies são registradas para Santa Catarina; estudos populacionais devem ser realizados para compreender melhor o estado de conservação das populações das espécies registradas.

\section{Agradecimentos}

Os autores agradecem à FAPESC - Fundação de Amparo à Pesquisa e Inovação do Estado de Santa Catarina, pelo financiamento do IFFSC, o que possibilitou as recentes coletas em todo o estado e aos revisores pelas valorosas contribuições.

\section{Referências}

Bittencourt, S.; Corte, A.P.D. \& Sanquetta, C.R. 2004. Estrutura da Comunidade de Pteridophyta em uma Floresta Ombrófila Mista, Sul do Paraná, Brasil. Silva Lusitana 12: 243-254.

Blume, M.; Fleck, R. \& Schmitt, J.L. 2010. Riqueza e composição de filicíneas e licófitas em um hectare de Floresta Ombrófila Mista no Rio Grande do Sul, Brasil. Revista Brasileira de Biociências 8 (4): 336-341.

Dittrich, V.A.O.; Kozera, C. \& Menezes-Silva, S. 1999. Levantamento florístico dos epífitos vasculares do Parque Barigüi, Curitiba, Paraná, Brasil. Iheringia, Série Botânica 52: 11-21.

Fidalgo, O. \& Bononi, V.L.R. 1984. Técnicas de coleta, preservação e herborização de material botânco. Instituto de Botânica, São Paulo.

Forzza, R.C.; Leitman, P.M.; Costa, A.F.; Carvalho Jr., A.A.; Peixoto, A.L.; Walter, B.M.T.; Bicudo, C.; Zappi, D.; Costa, D.P.; Lleras, E.; Martinelli, G.; Lima, H.C.; Prado, J.; Stehmann, J.R.; Baumgratz, J.F.A.; Pirani,
J.R.; Sylvestre, L.; Maia, L.C.; Lohmann, L.G.; Queiroz, L.P.; Silveira, M.; Coelho, M.N.; Mamede, M.C.; Bastos, M.N.C.; Morim, M.P.; Barbosa, M.R.; Menezes, M.; Hopkins, M.; Secco, R.; Cavalcanti, T.B.; Souza, V.C. 2010. Catálogo de Plantas e Fungos do Brasil. Instituto de Pesquisas Jardim Botânico do Rio de Janeiro / Andréa Jakobsson Estúdio, Rio de Janeiro.

Fuchs-Eckert, H.P. 1986. Isoetáceas. In: Reitz, R. Flora Ilustrada Catarinense. Herbário Barbosa Rodrigues, Itajaí.

Gasper, A.L. de \& Sevegnani, L. 2010. Lycophyta e samambaias do Parque Nacional da Serra do Itajaí, Vale do Itajaí, Santa Catarina, Brasil. Hoehnea 37 (4): 755-767.

Gasper, A.L. de; Sevegnani, L.; Vibrans, A.C.; Uhlmann, A.; Lingner, D.V.; Verdi, M.; Dreveck, S.; Stival-Santos, A.; Brogni, E.; Schmitt, R. \& Klemz, G. 2011. Inventário de Dicksonia sellowiana Hook. em Santa Catarina. Acta Botanica Brasilica 25(4): 776-784.

Hortal, J., Jiménez-Valverde, A. Gómez, J.F., Lobo, J.M. \& Baselga, A. 2008. Historical bias in biodiversity inventories affects the observed environmental niche of the species. Oikos 117: 847-858.

IBGE. 1992. Manual técnico da vegetação brasileira. Rio de Janeiro. IBGE.

Klein, R.M. 1972. Árvores nativas da Floresta Subtropical do Alto Uruguai. Sellowia 24: 9-62.

Klein, R.M. 1978. Mapa fitogeográfico do Estado de Santa Catarina. In Reitz, R. Flora Ilustrada Catarinense. Herbário Barbosa Rodrigues, Itajaí.

Klein, R.M. 1980a. Ecologia da flora e vegetação do Vale do Itajaí. Sellowia 31: 1-165.

Klein, R.M. 1980b. Ecologia da flora e vegetação do Vale do Itajaí (continuação). Sellowia 32: 165-389.

Klein, R.M. 1984. Aspectos Dinâmicos da Vegetação do Sul Do Brasil. Sellowia 36: 5-54

Kluge, J. \& Kessler, M. 2006. Fern endemism and its correlates: contribution from an elevational transect in Costa Rica. Diversity and Distributions 12 (5): 535-545.

Kozera, C.; Dittrich, V.A.O. \& Silva, S.M. 2006. Composição florística da floresta ombrófila mista montana do Parque Municipal do Barigüi, Curitiba, PR. Floresta 36(1): 45-58.

Kramer, K.U. \& Green. P.S. 1990. The families and genera of vascular plants - Pteridophytes and Gymnosperms. Ed. K. Kubitzki. SpringerVerlag Wien.

Lehn, C.R.; Leuchtenberger, C. \& Hansen, M.A.F. 2009. Pteridóftas ocorrentes em dois remanescentes de Floresta Estacional Decidual no Vale do Taquari, Estado do Rio Grande do Sul, Brasil. Iheringia, Sér. Bot 64(2): 23-31.

Leite, P.F. \& Klein, R.M. 1990. Geografia do Brasil. Região Sul. Rio de Janeiro, IBGE. 
Mantovani, M. 2004. Caracterização de populações naturais de Xaxim (Dicksonia sellowiana (Presl.) Hooker), em diferentes condições edafo-climáticas no Estado de Santa Catarina. Dissertação de Mestrado. Universidade Federal de Santa Catarina.

Martinelli, G. 2007. Mountain biodiversity in Brazi. Revista Brasileira de Botânica 30: 587-597.

Ministério do Meio Ambiente. 2008. Instrução Normativa No - 6, de 23 de Setembro de 2008.

Moran, R.C. 1995. The importance of mountains to pteridophytes, with emphasis on neotropical montane forests. Pp. 359-363. In: Churchill, S.P. Biodiversity and Conservation of Neotropical Montane Forest. New York, The New York Botanical Garden.

Moran, R.C. \& Smith, A.R. 2001. Phytogeographic relationships between neotropical and African-Madagascan pteridophytes. Brittonia 53(2): 304-351.

Moran, RC. 2008. Diversity, biogeography, and floristics. Pp. 367-394. In Ranker, T.A. \& Haufler, C.H. (Eds). Biology and evolution of ferns and lycophytes. New York, Cambridge University Press.

Morellato, L.P.C., \& Haddad, C.F.B. 2000. Introduction: The Brazilian Atlantic Forest. Biotropica 32 (4B): 786-792.

Nimer, E. 1971. Climatologia da Região Sul do Brasil. Revista Brasileira de Geografia 33(4): 3-65.

Page, C.N. 1979a. Experimental aspects of fern ecology. Pp. 551-589 In: Dyer, A.F. Experimental biology of ferns. London, Academic Press London.

Page, C.N. 1979b. The diversity of ferns: an ecological perspective. Pp. 9-56. In: Dyer, A.F. Experimental biology of ferns. London, Academic Press London.

Parris, B.S. 2001. Circum-Antarctic continental distribution patterns in pteridophyte species. Brittonia 53(2): 270-283.

Prado, J. \& Sylvestre, L.S. 2010. Pteridófitas. Pp. 522-566. In: Forzza, R.C.; Leitman, P.M.; Costa, A.F.; Carvalho Jr., A.A.; Peixoto, A.L.; Walter, B.M.T.; Bicudo, C.; Zappi, D.; Costa, D.P.; Lleras, E.; Martinelli, G.; Lima, H.C.; Prado, J.; Stehmann, J.R.; Baumgratz, J.F.A.; Pirani, J.R.; Sylvestre, L.; Maia, L.C.; Lohmann, L.G.; Queiroz, L.P.; Silveira, M.; Coelho, M.N.; Mamede, M.C.; Bastos, M.N.C.; Morim, M.P.; Barbosa, M.R.; Menezes, M.; Hopkins, M.; Secco, R.; Cavalcanti, T.B.; Souza, V.C. Catálogo de Plantas e Fungos do Brasil. Rio de Janeiro, Instituto de Pesquisas Jardim Botânico do Rio de Janeiro.

Pryer, K.M.; Schneider, H.; Smith, A.R.; Cranfill, R.B.; Wolf, P.G.; Hunt, J.S. \& Sipes, S.D. 2001. Horsetails and ferns are a monophyletic group and the closest living relatives to seed plants. Nature 409(6820): 618-622.

Pryer, K.M.; Schuettpelz, E.; Wolf, P.G.; Schneider, H.; Smith, A.R. \& Cranfill, R.B. 2004. Phylogeny and evolution of ferns (monilophytes) with a focus on the early leptosporangiate divergences. American Journal of Botany 91(10): 1582-1598.

Reitz, R. 1965. Plano de coleção. In: Reitz, R. Flora Ilustrada Catarinense. Herbário Barbosa Rodrigues, Itajaí.

Salino, A. \& Almeida, T.E. 2009. Pteridófitas. Pp 19-25. In: Stehmann, J.R.; Forzza, R.C.; Salino, A.; Sobral, M.; Costa, D.P. \&. Kamino, L.H.Y. Plantas da Floresta Atlântica. Rio de Janeiro: Instituto de Pesquisas Jardim Botânico do Rio de Janeiro.

Sampaio, M.B. \& Guarino, E.S.G. 2007. Efeitos do pastoreio de bovinos na estrutura populacional de plantas em fragmentos de Floresta Ombrófila Mista. Revista Árvore 31: 1035-1046.

Sastre, P. \& Lobo, J. 2009. Taxonomist survey biases and the unveiling of biodiversity patterns. Biological Conservation 142(2): 462-467.

Schneider, H.; Schuettpelz, E.; Pryer, K.M.; Cranfill, R.B.; Magallón, S. \& Lupia, R. 2004. Ferns diversified in the shadow of angiosperms. Nature 428(6982): 553-557.

Sehnem, A., 1967a. Maratiáceas. In: Reitz, R. Flora Ilustrada Catarinense. Herbário Barbosa Rodrigues, Itajaí.
Sehnem, A., 1967b. Osmundáceas. In: Reitz, R. Flora Ilustrada Catarinense. Herbário Barbosa Rodrigues, Itajaí.

Sehnem, A., 1967c. Vitariáceas. In: Reitz, R. Flora Ilustrada Catarinense. Herbário Barbosa Rodrigues, Itajaí.

Sehnem, A., 1968a. Aspleniáceas. In: Reitz, R. Flora Ilustrada Catarinense. Herbário Barbosa Rodrigues, Itajaí.

Sehnem, A., 1968b. Blecnáceas. In: Reitz, R. Flora Ilustrada Catarinense. Herbário Barbosa Rodrigues, Itajaí.

Sehnem, A., 1970a. Gleicheniáceas. In: Reitz, R. Flora Ilustrada Catarinense. Herbário Barbosa Rodrigues, Itajaí.

Sehnem, A., 1970b. Polipodiáceas. In: Reitz, R. Flora Ilustrada Catarinense. Herbário Barbosa Rodrigues, Itajaí.

Sehnem, A., 1971. Himenofiláceas. In: Reitz, R. Flora Ilustrada Catarinense. Herbário Barbosa Rodrigues, Itajaí.

Sehnem, A., 1972. Pteridaceae. In: Reitz, R. Flora Ilustrada Catarinense. Herbário Barbosa Rodrigues, Itajaí.

Sehnem, A., 1974. Esquizeaceás. In: Reitz, R. Flora Ilustrada Catarinense. Herbário Barbosa Rodrigues, Itajaí.

Sehnem, A., 1978. Ciateáceas. In: Reitz, R. Flora Ilustrada Catarinense. Herbário Barbosa Rodrigues, Itajaí.

Sehnem, A., 1979a. Aspidiáceas. In: Reitz, R. Flora Ilustrada Catarinense. Herbário Barbosa Rodrigues, Itajaí.

Sehnem, A., 1979b. Davaliáceas. In: Reitz, R. Flora Ilustrada Catarinense. Herbário Barbosa Rodrigues, Itajaí.

Sehnem, A., 1979c. Marsileáceas. In: Reitz, R. Flora Ilustrada Catarinense. Herbário Barbosa Rodrigues, Itajaí.

Sehnem, A., 1979d. Ofioglossáceas. In: Reitz, R. Flora Ilustrada Catarinense. Herbário Barbosa Rodrigues, Itajaí.

Sehnem, A., 1979e. Parkeriáceas. In: Reitz, R. Flora Ilustrada Catarinense. Herbário Barbosa Rodrigues, Itajaí.

Sehnem, A., 1979f. Psilotáceas. In: Reitz, R. Flora Ilustrada Catarinense. Herbário Barbosa Rodrigues, Itajaí.

Sehnem, A., 1979g. Salviniáceas. In: Reitz, R. Flora Ilustrada Catarinense. Herbário Barbosa Rodrigues, Itajaí.

Sehnem, A., 1984. Equisetáceas. In: Reitz, R. Flora Ilustrada Catarinense. Herbário Barbosa Rodrigues, Itajaí.

Senna, R.M. \& Waechter, J.L. 1997. Pteridófitas de uma floresta com araucária. 1. Formas biológicas e padrões de distribuição geográfica. Iheringia, Série Botânica 48: 41-58.

Smith, A.R., Pryer, K.M., Schuettpelz, E., Korall, P., Schneider, H. \& Wolf, P.G. 2006. A classification for extant ferns. Taxon 55: 705-731.

Sylvestre, L.S. 2001. Revisão taxonômica das espécies da família Aspleniaceae A. B. Frank ocorrentes no Brasil. Tese de doutorado. Universidade de São Paulo.

Vibrans, A.C., Sevegnani, L., Lingner, D.V., Gasper, A.L. de \& Sabbagh, S. 2010. Inventário florístico florestal de Santa Catarina (IFFSC): aspectos metodológicos e operacionais. Pesquisa Florestal Brasileira 30(64): 291-302.

Vibrans, A.C.; Sevegnani, L.; Uhlmann, A.; Schorn, L.A.; Sobral, M.; Gasper, A.L. de; Lingner, D.V.; Brogni, E.; Klemz, G.; Godoy, M.B. \& Verdi, M. 2011. Structure of mixed ombrophyllous forests with Araucaria angustifolia (Araucariaceae) under external stress in Southern Brazil. Revista de Biologia Tropical 59(3): 1371-1387.

Wilcove, D.S.; Rothstein, D.; Dubow, J. \& Phillips, A. 1998. Quantifying threats to imperiled species in the United States. BioScience 48(8): 607-615.

Windisch, P.G. 1992. Pteridófitas da Região Norte-Ocidental do Estado de São Paulo - Guia para excursões. 2. ed. São José do Rio Preto, Editora Universitária-UNESP.

Windisch, P.G. 2002. Pteridófitas do Brasil: Diversidade Decrescente. Biodiversidade, Conservação e Uso Sustentável da Flora do Brasil 1: 196-198. 